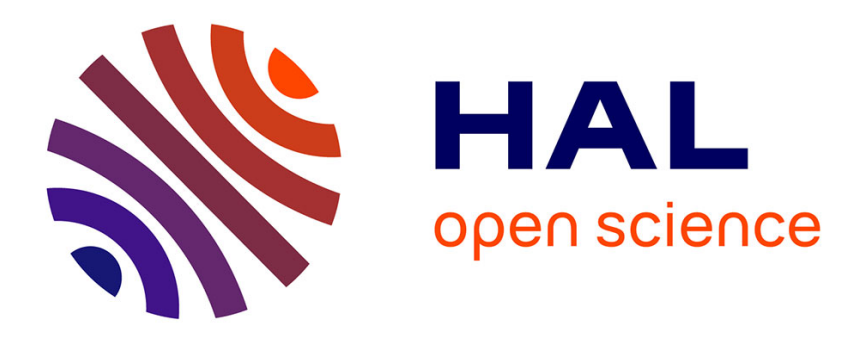

\title{
Well-posedness and stability results for laminated Timoshenko beams with interfacial slip and infinite memory
}

Aissa Guesmia

\section{- To cite this version:}

Aissa Guesmia. Well-posedness and stability results for laminated Timoshenko beams with interfacial slip and infinite memory. IMA Journal of Mathematical Control and Information, 2019, 00, pp.1 - 51. 10.1093/imamci/dnz002 . hal-02891558

\section{HAL Id: hal-02891558 \\ https://hal.science/hal-02891558}

Submitted on 7 Jul 2020

HAL is a multi-disciplinary open access archive for the deposit and dissemination of scientific research documents, whether they are published or not. The documents may come from teaching and research institutions in France or abroad, or from public or private research centers.
L'archive ouverte pluridisciplinaire HAL, est destinée au dépôt et à la diffusion de documents scientifiques de niveau recherche, publiés ou non, émanant des établissements d'enseignement et de recherche français ou étrangers, des laboratoires publics ou privés. 


\title{
Well-posedness and stability results for laminated Timoshenko beams with interfacial slip and infinite memory
}

\author{
Aissa Guesmia* \\ Institut Elie Cartan de Lorraine, UMR 7502, Université de Lorraine, 3 Rue Augustin Fresnel, \\ BP 45112, 57073 Metz Cedex 03, France and Department of Mathematics and Statistics, \\ King Fahd University of Petroleum and Minerals, Dhahran 31261, Saudi Arabia \\ *Corresponding author. Email: aissa.guesmia@univ-lorraine.fr
}

[Received on 3 August 2018; revised on 27 October 2018; accepted on 27 December 2018]

\begin{abstract}
The model under consideration in this paper describes a vibrating structure of an interfacial slip and consists of three coupled hyperbolic equations in one-dimensional bounded interval under mixed homogeneous Dirichlet-Neumann boundary conditions. The first two equations are related to Timoshenko-type systems and the third one is subject to the dynamics of the slip. The main problem we discuss here is stabilizing the system by a viscoelastic damping generated by an infinite memory and acting only on one equation. First, we prove the existence, uniqueness and regularity of solutions using the semigroup theory. After that, we combine the energy method and the frequency domain approach to show that the infinite memory is capable alone to guarantee the strong and polynomial stability of the model, that is bringing it back to its equilibrium state with a decay rate of type $t^{-d}$, where $d$ is a positive constant depending on the regularity of initial data. Moreover, we prove that, when the infinite memory is effective on the first equation, the model is not exponentially stable independently of the values of the parameters. However, when the infinite memory is effective on the second or the third equation, we prove that the exponential stability is equivalent to the equality between the three speeds of wave propagations. An extension of our results to the frictional damping case is also given. Our results improve and extend some existing results in the literature subject to other types of controls.
\end{abstract}

Keywords: Timoshenko beam with interfacial slip; infinite memory; well-posedness; asymptotic behavior; energy method; frequency domain approach.

\section{Introduction}

The structures known under the name laminated Timoshenko beams are composed of two layered identical beams of uniform thickness and attached together on top of each other subject to transversal and rotational vibrations and taking account the longitudinal displacement. An adhesive layer of small thickness is bonding the two adjoining surfaces and creating a restoring force being proportional to the amount of slip and producing a damping. These structures are used in many practical fields; see, for example, Lo \& Tatar (2015) for more details. When the longitudinal displacement (slip) is ignored, the laminated Timoshenko beams are reduced to the well-known Timoshenko beams (Timoshenko, 1921). But in many practical cases, the slip is present and, logically, it cannot be avoided. During the past few years, these structures were the subject of several studies in the literature recovering well-posedness and stability by adding some kinds of controls. The obtained stability results depend on the nature, number and position of the controls and some relations between the parameters (constants) of the model. The first researchers have been forced to stabilize the system by additional boundary controls. After that, 
some other internal controls were considered. Let us mention here some of these results related to our objectives in this paper.

Mathematically, a model of laminated Timoshenko beams of length 1 and with interfacial slip based on the Timoshenko theory is given by the system (see Hansen, 1994 and Hansen \& Spies, 1997)

$$
\left\{\begin{array}{l}
\rho w_{t t}+G\left(\psi-w_{x}\right)_{x}=0 \\
I_{\rho}(3 s-\psi)_{t t}-G\left(\psi-w_{x}\right)-D(3 s-\psi)_{x x}=0 \\
3 I_{\rho} s_{t t}+3 G\left(\psi-w_{x}\right)+4 \gamma s+4 \beta s_{t}-3 D s_{x x}=0
\end{array}\right.
$$

where the subscripts $x$ and $t$ denote the derivative with respect to space and time variables $x$ and $t$, respectively, $x \in(0,1)$ and $t>0$, combining with some initial data and boundary conditions at $x=0$ and $x=1$. The parameters $\rho, G, I_{\rho}, D, \gamma$ and $\beta$ are positive constants and denote the density, shear stiffness, mass moment of inertia, flexural rigidity, adhesive stiffness and adhesive damping parameter, respectively. The functions $w=(x, t)$ and $\psi=(x, t)$ represent the transverse displacement and rotation angle, respectively, and the function $s=s(x, t)$ is proportional to the amount of slip along the interface, so the third equation in (1.1) describes the dynamics of the slip and contains the internal frictional damping $4 \beta s_{t}$. Without loss of generality, the length of the beam is assumed to be equal to 1 instead of $l$. When the third equation in (1.1) is not taken in consideration (i.e. $s=0$ ), that is (1.1) is reduced to Timoshenko (1921) beams, the stability question was widely treated in a huge number of works; see, for example, Cavalcanti et al. (2014) and Guesmia et al. (2012) and the references therein.

Wang et al. (2005) considered the change of variable given by the effective rotation angle $\xi=3 s-\psi$ and proved the exponential stability through mixed homogeneous Dirichlet-Neumann boundary conditions and two boundary controls at $x=1$ provided that the speeds of wave propagations of the first two equations (transverse displacement and rotation angle) are different, that is

$$
\sqrt{\frac{G}{\rho}} \neq \sqrt{\frac{D}{I_{\rho}}} .
$$

It was also proved in Wang et al. (2005) that the frictional damping $4 \beta s_{t}$ created by the interfacial slip alone is strong enough to stabilize asymptotically the structure (with a decay rate of polynomial type) but it is not able to stabilize the structure exponentially. The same exponential stability result of Wang et al. (2005) was proved in Cao et al. (2007) for the same model but through two boundary controls at $x=0$ and $x=1$. The exponential stability result of Wang et al. (2005) was improved in Tatar (2015) by dropping some conditions on the parameters. Lo \& Tatar (2016b) proved that the exponential stability of (1.1) holds if the boundary controls are replaced by a frictional damping acting on the first equation (transverse displacement). Raposo (2016) proved that the exponential stability holds without any restriction on the parameters if the first two equations in (1.1) are also damped via frictional dampings. Recently, Raposo et al. (2017) proved that, without any restriction on the parameters, the polynomial stability of (1.1) holds under additional three dynamic boundary conditions at $l$, where $l$ is the length of the beam. For the stability of laminated beams with Cattaneo's or Fourier's type heat conduction, we refer the readers to Alves et al. (2016) and Liu \& Zhao (2017).

The viscoelastic dampings, which are represented by a memory term in the form of a convolution, may be caused by the utilization of special materials able to drive the structures to rest in a very fast manner without any other kinds of controls. These materials are useful if there is no change of frequency or temperature in the structures. For more details, see, for example, Beards \& Imam (1978), Lo \& Tatar 
(2015) and Lo \& Tatar (2016b). The stability of (1.1) under viscoelastic dampings was treated in Lo \& Tatar (2015), Lo \& Tatar (2016a) and Lo \& Tatar (2016b). Namely, provided that $G$ is small enough and (1.2) is not satisfied, the exponential stability of (1.1) was proved in Lo \& Tatar (2015), Lo \& Tatar (2016a) and Lo \& Tatar (2016b) by adding viscoelastic dampings represented by finite memories of the form

$$
\int_{0}^{t} h_{1}(t-\tau) w_{x x}(x, \tau) \mathrm{d} \tau, \quad \int_{0}^{t} h_{2}(t-\tau)(3 s-\psi)_{x x}(x, \tau) \mathrm{d} \tau \quad \text { and } \quad \int_{0}^{t} h_{3}(t-\tau) s_{x x}(x, \tau) \mathrm{d} \tau
$$

with $h_{1}=h_{3}=0$ in Lo \& Tatar (2015), $h_{1}=0$ (and $\beta=0$ ) in Lo \& Tatar (2016a) and $h_{3}=0$ in Lo \& Tatar (2016b), where $h_{j}: \mathbb{R}_{+} \rightarrow \mathbb{R}_{+}, j=1,2,3$ are differentiable, non-increasing, integrable on $\mathbb{R}_{+}$and satisfying, for some positive constants $\beta_{j}$ and $\alpha_{j}$,

$$
-\beta_{j} h_{j} \leq h_{j}^{\prime} \leq-\alpha_{j} h_{j}, \quad j=1,2,3
$$

For the stability of Bresse (1859) systems with infinite memories, we refer the readers to Guesmia (2017a,b), Guesmia \& Kafini (2015) and Guesmia \& Kirane (2016) and the references therein. The Bresse systems are similar to (1.1) and known as the circular arch problem.

From the cited results above, we see that the exponential and/or polynomial stability has been proved via at least two controls and/or under some restrictions on the parameters. On the other hand, the speeds of the wave propagations of the last two equations in (1.1) (rotation angle and amount of slip) are always assumed being the same (both are equal to $\sqrt{\frac{D}{I_{\rho}}}$ ). Therefore, a natural question that can be asked is what is the control that can guarantee alone the strong and/or polynomial and/or exponential stability of the system? And what is the minimum of restrictions on the parameters that are needed? We propose investigating the case where a unique viscoelastic control represented by an infinite memory is considered and no other internal or boundary control is present. More precisely, we study, in this paper, the well-posedness and the stability of three structures with interfacial slip and infinite memory effective only on one equation. The first case is when the memory is working on the transverse displacement

$$
\left\{\begin{array}{l}
\rho_{1} \varphi_{t t}+k\left(u-\varphi_{x}\right)_{x}+\int_{0}^{\infty} g(s) \varphi_{x x}(x, t-s) \mathrm{d} s=0 \\
\rho_{2}(3 v-u)_{t t}-b(3 v-u)_{x x}-k\left(u-\varphi_{x}\right)=0 \\
\tilde{\rho}_{3} v_{t t}-\tilde{k}_{0} v_{x x}+3 k\left(u-\varphi_{x}\right)+4 \tilde{\delta} v=0
\end{array}\right.
$$

with boundary conditions

$$
\varphi_{x}(0, t)=\varphi(1, t)=u(0, t)=u_{x}(1, t)=v(0, t)=v_{x}(1, t)=0
$$

and initial data

$$
(\varphi(x,-t), u(x, 0), v(x, 0))=\left(\varphi_{0}(x, t), u_{0}(x), v_{0}(x)\right)
$$

and

$$
\left(\varphi_{t}(x, 0), u_{t}(x, 0), v_{t}(x, 0)\right)=\left(\varphi_{1}(x), u_{1}(x), v_{1}(x)\right)
$$


The second case is when the memory is working on the rotation angle

$$
\left\{\begin{array}{l}
\rho_{1} \varphi_{t t}+k\left(u-\varphi_{x}\right)_{x}=0 \\
\rho_{2}(3 v-u)_{t t}-b(3 v-u)_{x x}-k\left(u-\varphi_{x}\right)+\int_{0}^{\infty} g(s)(3 v(x, t-s)-u(x, t-s))_{x x} \mathrm{~d} s=0, \\
\tilde{\rho}_{3} v_{t t}-\tilde{k}_{0} v_{x x}+3 k\left(u-\varphi_{x}\right)+4 \tilde{\delta} v=0
\end{array}\right.
$$

with boundary conditions (1.6) and initial data (1.8) and

$$
(\varphi(x, 0), u(x,-t), v(x,-t))=\left(\varphi_{0}(x, 0), u_{0}(x, t), v_{0}(x, t)\right)
$$

The third case is when the memory is working on the amount of slip

$$
\left\{\begin{array}{l}
\rho_{1} \varphi_{t t}+k\left(u-\varphi_{x}\right)_{x}=0 \\
\rho_{2}(3 v-u)_{t t}-b(3 v-u)_{x x}-k\left(u-\varphi_{x}\right)=0 \\
\tilde{\rho}_{3} v_{t t}-\tilde{k}_{0} v_{x x}+3 k\left(u-\varphi_{x}\right)+4 \tilde{\delta} v+\int_{0}^{\infty} \tilde{g}(s) v_{x x}(x, t-s) \mathrm{d} s=0
\end{array}\right.
$$

with boundary conditions (1.6) and initial data (1.8) and

$$
(\varphi(x, 0), u(x, 0), v(x,-t))=\left(\varphi_{0}(x), u_{0}(x), v_{0}(x, t)\right)
$$

Here $(x, t) \in(0,1) \times \mathbb{R}_{+}, \varphi=\varphi(x, t)$ is the transverse displacement; $u=u(x, t)$ represents the rotation angle; $v=v(x, t)$ is proportional to the amount of slip along the interface; $\rho_{1}, \rho_{2}, \tilde{\rho}_{3}, k$, $b, \tilde{k}_{0}$ and $\tilde{\delta}$ are positive constants; $g, \tilde{g}: \mathbb{R}_{+} \rightarrow \mathbb{R}_{+}$are given relaxation functions satisfying some hypotheses and $\varphi_{0}, u_{0}, v_{0}, \varphi_{1}, u_{1}$ and $v_{1}$ are given functions belonging to a suitable Hilbert space. The systems (1.5), (1.9) and (1.11) are given in more general form than the one considered in the literature, since the speeds of wave propagations of the last two equations are not necessarily equal and no frictional damping is present in the last equation. Our objectives in this paper is proving the well-posedness of these three systems and establishing their stability in terms of the parameters and the relaxation functions.

For the stability, first, we show that (1.9) and (1.11) are strongly and polynomially stable without any restrictions on the parameters, where the decay rate for classical solutions is arbitrarily close to $t^{-\frac{1}{10}}$. Moreover, we prove that (1.9) and (1.11) are exponentially stable if and only if the three speeds of wave propagations are equal. Second, we prove that the strong and polynomial stability of (1.5) (with a decay rate for classical solutions arbitrarily close to $t^{-\frac{1}{4}}$ ) hold provided that the speeds of wave propagations of the last two equations in (1.5) are equal or $\tilde{\delta}$ does not belong to a given sequence of real numbers. Third, we prove that (1.5) is not exponentially stable whichever the values of the parameters. Finally, we give an extension to the case where the infinite memory is replaced by a frictional damping by proving that our results are still satisfied and the polynomial decay rate for classical solutions is arbitrarily close to $t^{-\frac{1}{8}}$ and $t^{-\frac{1}{2}}$ instead of $t^{-\frac{1}{10}}$ and $t^{-\frac{1}{4}}$, respectively.

The proof of the well-posedness is based on the semigroup theory. However, the stability results are proved using the energy method combined with the frequency domain approach. 
The paper is organized as follows. In Section 2, we consider some assumptions on the relaxation functions and prove the well-posedness. In Sections 3, 4, 5 and 6, we prove our strong, exponential, polynomial and lack of exponential stability, respectively. In Section 7, we give an extension of our results to the case of frictional damping. We end our paper by giving some general comments and issues in Section 8.

\section{Setting of the semigroup}

To simplify the mathematical study of our systems (1.5), (1.9) and (1.11), we put

$$
\left\{\begin{array}{l}
\rho_{3}=\frac{1}{9} \tilde{\rho}_{3}, \quad k_{0}=\frac{1}{9} \tilde{k}_{0}, \quad \delta=\frac{4}{9} \tilde{\delta}, \quad g=\frac{1}{9} \tilde{g} \\
w=-3 v, \quad \psi=3 v-u, \quad w_{0}=-3 v_{0}, \quad w_{1}=-3 v_{1}, \quad \psi_{0}=3 v_{0}-u_{0}, \quad \psi_{1}=3 v_{1}-u_{1}
\end{array}\right.
$$

Then the systems (1.5), (1.9) and (1.11) are reduced to

$$
\left\{\begin{array}{l}
\rho_{1} \varphi_{t t}-k\left(\varphi_{x}+\psi+w\right)_{x}+F_{1}=0 \\
\rho_{2} \psi_{t t}-b \psi_{x x}+k\left(\varphi_{x}+\psi+w\right)+F_{2}=0 \\
\rho_{3} w_{t t}-k_{0} w_{x x}+k\left(\varphi_{x}+\psi+w\right)+\delta w+F_{3}=0
\end{array}\right.
$$

where

$$
F_{2}=F_{3}=0 \quad \text { and } \quad F_{1}(x, t)=\int_{0}^{\infty} g(s) \varphi_{x x}(x, t-s) \mathrm{d} s
$$

in case (1.5),

$$
F_{1}=F_{3}=0 \quad \text { and } \quad F_{2}(x, t)=\int_{0}^{\infty} g(s) \psi_{x x}(x, t-s) \mathrm{d} s
$$

in case (1.9) and

$$
F_{1}=F_{2}=0 \quad \text { and } \quad F_{3}(x, t)=\int_{0}^{\infty} g(s) w_{x x}(x, t-s) \mathrm{d} s
$$

in case (1.11). The boundary conditions (1.6) become

$$
\varphi_{x}(0, t)=\varphi(1, t)=\psi(0, t)=\psi_{x}(1, t)=w(0, t)=w_{x}(1, t)=0, \quad t \in \mathbb{R}_{+},
$$

and the initial data are now

$$
\left\{\begin{array}{ll}
(\varphi(x,-t), \psi(x, 0), w(x, 0))=\left(\varphi_{0}(x, t), \psi_{0}(x), w_{0}(x)\right) & \text { in case }(2.2), \\
(\varphi(x, 0), \psi(x,-t), w(x, 0))=\left(\varphi_{0}(x), \psi_{0}(x, t), w_{0}(x)\right) & \text { in case }(2.3), \\
(\varphi(x, 0), \psi(x, 0), w(x,-t))=\left(\varphi_{0}(x), \psi_{0}(x), w_{0}(x, t)\right) & \text { in case }(2.4),
\end{array} \quad x \in(0,1), t \in \mathbb{R}_{+} .\right.
$$


To rewrite our system (2.1) with (2.5) and (2.6) in an abstract first-order system in a suitable Hilbert space $\mathcal{H}$, we consider the functional $\eta$ introduced in Dafermos (1970) and its initial data $\eta_{0}$ given by

$$
\begin{cases}\eta(x, t, s)=f(x, t)-f(x, t-s), & x \in(0,1), s, t \in \mathbb{R}_{+}, \\ \eta_{0}(x, s)=f_{0}(x, 0)-f_{0}(x, s), & x \in(0,1), s \in \mathbb{R}_{+},\end{cases}
$$

where

$$
f=\left\{\begin{array}{ll}
\varphi & \text { in case (2.2), } \\
\psi & \text { in case (2.3), } \\
w & \text { in case (2.4) }
\end{array} \quad \text { and } \quad f_{0}= \begin{cases}\varphi_{0} & \text { in case (2.2) } \\
\psi_{0} & \text { in case (2.3) } \\
w_{0} & \text { in case (2.4) }\end{cases}\right.
$$

Then the integral term becomes

$$
\int_{0}^{\infty} g(s) f_{x x}(x, t-s) \mathrm{d} s=g_{0} f_{x x}(x, t)-\int_{0}^{\infty} g(s) \eta_{x x}(x, t, s) \mathrm{d} s,
$$

where

$$
g_{0}=\int_{0}^{\infty} g(s) \mathrm{d} s
$$

The functional $\eta$ satisfies

$$
\begin{cases}\eta_{t}(x, t, s)+\eta_{s}(x, t, s)-\tilde{f}(x, t)=0, & x \in(0,1), s, t>0 \\ \eta(x, 0, s)=\eta_{0}(x, s), & x \in(0,1), s \in \mathbb{R}_{+} \\ \eta(x, t, 0)=0, & x \in(0,1), t \in \mathbb{R}_{+}\end{cases}
$$

where the subscript $s$ denotes the derivative with respect to $s$,

$$
\tilde{f}= \begin{cases}\tilde{\varphi} & \text { in case (2.2), } \\ \tilde{\psi} & \text { in case (2.3), } \\ \tilde{w} & \text { in case (2.4) }\end{cases}
$$

Let

$$
\left\{\begin{array}{l}
U=(\varphi, \tilde{\varphi}, \psi, \tilde{\psi}, w, \tilde{w}, \eta) \\
U_{0}=\left(\varphi_{0}, \varphi_{1}, \psi_{0}, \psi_{1}, w_{0}, w_{1}, \eta_{0}\right)
\end{array}\right.
$$

Now, we can rewrite the system (2.1) with its initial data (2.6) in the following initial value problem:

$$
\left\{\begin{array}{l}
U_{t}(t)=\mathcal{A} U(t), \quad t>0 \\
U(0)=U_{0}
\end{array}\right.
$$


where $\mathcal{A}$ is a linear operator given by

$$
\mathcal{A} U=\left(\begin{array}{c}
\tilde{\varphi} \\
\frac{k}{\rho_{1}}\left(\varphi_{x}+\psi+w\right)_{x} \\
\tilde{\psi} \\
\frac{1}{\rho_{2}}\left[b \psi_{x x}-k\left(\varphi_{x}+\psi+w\right)\right] \\
\tilde{w} \\
\frac{1}{\rho_{3}}\left[k_{0} w_{x x}-k\left(\varphi_{x}+\psi+w\right)-\delta w\right] \\
-\eta_{s}+\tilde{f}
\end{array}\right)+\mathcal{B} U,
$$

where

$$
\mathcal{B} U=\left(0,-\frac{g_{0}}{\rho_{1}} \varphi_{x x}+\frac{1}{\rho_{1}} \int_{0}^{\infty} g(s) \eta_{x x} \mathrm{~d} s, 0,0,0,0,0\right)^{T}
$$

in case (2.2),

$$
\mathcal{B} U=\left(0,0,0,-\frac{g_{0}}{\rho_{2}} \psi_{x x}+\frac{1}{\rho_{2}} \int_{0}^{\infty} g(s) \eta_{x x} \mathrm{~d} s, 0,0,0\right)^{T}
$$

in case (2.3) and

$$
\mathcal{B} U=\left(0,0,0,0,0,-\frac{g_{0}}{\rho_{3}} w_{x x}+\frac{1}{\rho_{3}} \int_{0}^{\infty} g(s) \eta_{x x} \mathrm{~d} s, 0\right)^{T}
$$

in case (2.4).

To define the energy Hilbert space $\mathcal{H}$, we consider the standard $L^{2}(0,1)$ space with its classical scalar product $\langle\cdot, \cdot\rangle$ and its norm $\|\cdot\|$. We consider also the phase Hilbert space

$$
\tilde{H}= \begin{cases}H_{1} & \text { in case (2.2), } \\ H_{0} & \text { in cases(2.3) and(2.4) }\end{cases}
$$

where

$$
H_{1}=\left\{v \in H^{1}(0,1): v(1)=0\right\} \quad \text { and } \quad H_{0}=\left\{v \in H^{1}(0,1): v(0)=0\right\} .
$$

We also introduce the Hilbert space

$$
L_{g}=\left\{v: \mathbb{R}_{+} \rightarrow \tilde{H}, \int_{0}^{\infty} g(s)\left\|v_{x}(s)\right\|^{2} \mathrm{~d} s<\infty\right\}
$$


equipped with the inner product

$$
\langle\eta, \tilde{\eta}\rangle_{L_{g}}=\int_{0}^{\infty} g(s)\left\langle\eta_{x}(s), \tilde{\eta}_{x}(s)\right\rangle \mathrm{d} s .
$$

The energy space is given by

$$
\mathcal{H}=H_{1} \times L^{2}(0,1) \times H_{0} \times L^{2}(0,1) \times H_{0} \times L^{2}(0,1) \times L_{g}
$$

equipped with the inner product, for any

$$
\begin{aligned}
U_{1}= & \left(\varphi_{1}, \tilde{\varphi}_{1}, \psi_{1}, \tilde{\psi}_{1}, w_{1}, \tilde{w}_{1}, \eta_{1}\right), U_{2}=\left(\varphi_{2}, \tilde{\varphi}_{2}, \psi_{2}, \tilde{\psi}_{2}, w_{2}, \tilde{w}_{2}, \eta_{2}\right) \in \mathcal{H}, \\
\left\langle U_{1}, U_{2}\right\rangle_{\mathcal{H}}= & k\left\langle\left(\varphi_{1 x}+\psi_{1}+w_{1}\right),\left(\varphi_{2 x}+\psi_{2}+w_{2}\right)\right\rangle+b\left\langle\psi_{1 x}, \psi_{1 x}\right\rangle+k_{0}\left\langle w_{1 x}, w_{2 x}\right\rangle \\
+ & \delta\left\langle w_{1}, w_{2}\right\rangle-g_{0}\left\langle f_{1 x}, f_{2 x}\right\rangle+\rho_{1}\left\langle\tilde{\varphi}_{1}, \tilde{\varphi}_{2}\right\rangle+\rho_{2}\left\langle\tilde{\psi}_{1}, \tilde{\psi}_{2}\right\rangle+\rho_{3}\left\langle\tilde{w}_{1}, \tilde{w}_{2}\right\rangle+\left\langle\eta_{1}, \eta_{2}\right\rangle_{L_{g}},
\end{aligned}
$$

where $f_{j}$ is defined in terms of $\psi_{j}, \varphi_{j}$ and $w_{j}, j=1,2$, as $f$ in (2.8). The homogeneous Dirichlet boundary conditions are guaranteed by the definition of $\mathcal{H}$, and the homogeneous Neumann ones are taken in consideration in the definition of $D(\mathcal{A})$ given by

$$
D(\mathcal{A})=\left\{U \in \mathcal{H}, \mathcal{A} U \in \mathcal{H}, \varphi_{x}(0)=\psi_{x}(1)=w_{x}(1)=\eta(s=0)=0\right\}
$$

To guarantee that $\mathcal{H}$ is a Hilbert space and get the well-posedness of (2.12), we consider the following hypothesis:

(H) Assume that the function $g: \mathbb{R}_{+} \rightarrow \mathbb{R}_{+}$is differentiable, non-increasing and integrable on $\mathbb{R}_{+}$ such that $g_{0}>0$ and there exists a positive constant $\tilde{k}$ satisfying, for any

$$
(\varphi, \psi, w) \in H_{1} \times H_{0} \times H_{0}
$$

the following inequality:

$$
\tilde{k}\left(\left\|\varphi_{x}\right\|^{2}+\left\|\psi_{x}\right\|^{2}+\left\|w_{x}\right\|^{2}\right) \leq k\left\|\varphi_{x}+\psi+w\right\|^{2}+b\left\|\psi_{x}\right\|^{2}+k_{0}\left\|w_{x}\right\|^{2}+\delta\|w\|^{2}-g_{0}\left\|f_{x}\right\|^{2}
$$

Moreover, assume that there exist positive constants $\beta_{1}$ and $\beta_{2}$ such that

$$
-\beta_{1} g \leq g^{\prime} \leq-\beta_{2} g
$$

Remark 1 1. Condition (2.20) holds with $\tilde{k}=\hat{k}-g_{0}$ if $g_{0}<\hat{k}$, where $\hat{k}$ is the biggest constant satisfying

$$
\hat{k}\left(\left\|\varphi_{x}\right\|^{2}+\left\|\psi_{x}\right\|^{2}+\left\|w_{x}\right\|^{2}\right) \leq k\left\|\varphi_{x}+\psi+w\right\|^{2}+b\left\|\psi_{x}\right\|^{2}+k_{0}\left\|w_{x}\right\|^{2}+\delta\|w\|^{2},
$$


for any $(\varphi, \psi, w) \in H_{1} \times H_{0} \times H_{0}$. Notice that, according to the considered homogeneous Dirichlet boundary conditions and Poincaré's inequality, when the right-hand side of (2.22) vanishes, we have $\varphi=\psi=w=0$ (even if $\delta=0$ ). So, under condition (2.20), $\mathcal{H}$ is a Hilbert space and its inner product generates a norm

$$
\|U\|_{\mathcal{H}}^{2}=\langle U, U\rangle_{\mathcal{H}}
$$

equivalent to the one of $\left[H^{1}(0,1) \times L^{2}(0,1)\right]^{3} \times L_{g}$.

2. By considering the particular choices $(\psi, w) \stackrel{g}{=}(0,0),(\varphi, w)=(0,0)$ and $(\varphi, \psi)=(0,0)$, we see that condition (2.20) leads to

$$
\begin{cases}k-g_{0} \geq \tilde{k} & \text { in case (2.2) } \\ b-g_{0} \geq \tilde{k} & \text { in case (2.3) } \\ k_{0}-g_{0} \geq \tilde{k} & \text { in case (2.4) }\end{cases}
$$

This ensures the hyperbolicity of (2.1).

3. Condition (2.21) implies that

$$
g(0) e^{-\beta_{1} s} \leq g(s) \leq g(0) e^{-\beta_{2} s}, \quad s \in \mathbb{R}_{+},
$$

so the typical functions $g$ satisfying (2.21) are

$$
g(s)=b_{1} e^{-b_{2} s},
$$

where $b_{1}$ and $b_{2}$ are positive constants.

4. If $g_{0}=0$, then $g \equiv 0$ and therefore (2.12) is a conservative system, that is $t \mapsto\|U(t)\|_{\mathcal{H}}^{2}$ is a constant function. Indeed, using (2.12), (2.13), (2.23) and a direct computation, we get, for any $U \in D(\mathcal{A})$,

$$
\left(\|U\|_{\mathcal{H}}^{2}\right)^{\prime}=2\left\langle U_{t}, U\right\rangle_{\mathcal{H}}=2\langle\mathcal{A} U, U\rangle_{\mathcal{H}}=-2 \int_{0}^{1} \int_{0}^{\infty} g(s) \eta_{x} \eta_{x s} \mathrm{~d} s \mathrm{~d} x=-\int_{0}^{1} \int_{0}^{\infty} g(s)\left(\eta_{x}^{2}\right)_{s} \mathrm{~d} s \mathrm{~d} x
$$

By integrating with respect to $s$ and noting that

$$
\eta(s=0)=0 \quad \text { and } \quad \lim _{\tau \rightarrow \infty} g(\tau)\left\|\eta_{x}(s=\tau)\right\|^{2}=0
$$

we find

$$
\left(\|U\|_{\mathcal{H}}^{2}\right)^{\prime}=\int_{0}^{\infty} g^{\prime}(s)\left\|\eta_{x}\right\|^{2} \mathrm{~d} s
$$

Notice that the left-hand inequality in (2.21) implies that the integral in (2.26) is bounded. So, if $g \equiv 0$, then (2.12) is a conservative system, that is

$$
\|U(t)\|_{\mathcal{H}}^{2}=\left\|U_{0}\right\|_{\mathcal{H}}^{2}, \quad t \in \mathbb{R}_{+}
$$


Our well-posedness results are presented in the following theorem:

Theorem 2.1 Assume that $(\mathbf{H})$ holds. Then, for any $m \in \mathbb{N}$ and $U_{0} \in D\left(\mathcal{A}^{m}\right)$, system (2.12) admits a unique solution $U$ satisfying

$$
U \in \cap_{j=0}^{m} C^{m-j}\left(\mathbb{R}_{+} ; D\left(\mathcal{A}^{j}\right)\right) .
$$

Proof. First, direct calculations give (as for (2.26))

$$
\langle\mathcal{A} U, U\rangle_{\mathcal{H}}=\frac{1}{2} \int_{0}^{\infty} g^{\prime}(s)\left\|\eta_{x}\right\|^{2} \mathrm{~d} s \leq 0 .
$$

Hence, $\mathcal{A}$ is a dissipative operator, since $g$ is non-increasing.

Next, we show that $0 \in \rho(\mathcal{A})$, where $\rho(\mathcal{A})$ is the resolvent set of $\mathcal{A}$, that is, for any $F=\left(f_{1}, \cdots, f_{7}\right) \in \mathcal{H}$, there exists $U \in D(\mathcal{A})$ satisfying

$$
\mathcal{A} U=F .
$$

Let us treat the case (2.3). The other two cases (2.2) and (2.4) can be treated exactly in the same way.

First, using (2.13) and (2.15), the first, third and fifth equations in (2.29) are equivalent to

$$
\tilde{\varphi}=f_{1}, \quad \tilde{\psi}=f_{3} \quad \text { and } \quad \tilde{w}=f_{5},
$$

and then

$$
\tilde{\varphi} \in H_{1} \quad \text { and } \quad \tilde{\psi}, \tilde{w} \in H_{0} .
$$

Second, using the second equality in (2.30), the last equation in (2.29) (with $\tilde{f}=\tilde{\psi}$ ) is reduced to

$$
\eta_{s}=f_{3}-f_{7}
$$

A direct integration proves that the unique solution $\eta$ of this equation satisfying $\eta(s=0)=0$ is given by

$$
\eta(s)=s f_{3}-\int_{0}^{s} f_{7}(\tau) \mathrm{d} \tau
$$

Because $g$ is integrable, $f_{3} \in H_{0}$ and $f_{7} \in L_{g}$, we see that $\eta_{s} \in L_{g}$ and $\eta(x=0)=0$. On the other hand, the right-hand inequality in (2.25) leads to

$$
s \mapsto s f_{3} \in L_{g}
$$

Moreover, by a direct integration, we remark that the right-hand inequality in (2.21) implies that

$$
\int_{\tau}^{\infty} g(s) \mathrm{d} s \leq \frac{1}{\beta_{2}} g(\tau), \quad \tau \in \mathbb{R}_{+},
$$


then exploiting this inequality, Fubini theorem and Hölder's inequality, we get

$$
\begin{aligned}
\int_{0}^{1} \int_{0}^{\infty} g(s)\left|\int_{0}^{s} f_{7 x}(\tau) \mathrm{d} \tau\right|^{2} \mathrm{~d} s \mathrm{~d} x & \leq \int_{0}^{\infty} g(s) \int_{0}^{s}\left\|f_{7 x}(\tau)\right\|^{2} \mathrm{~d} \tau \mathrm{d} s \\
& \leq \int_{0}^{\infty}\left\|f_{7 x}(\tau)\right\|^{2} \int_{\tau}^{\infty} g(s) \mathrm{d} s \mathrm{~d} \tau \\
& \leq \frac{1}{\beta_{2}} \int_{0}^{\infty} g(\tau)\left\|f_{7 x}(\tau)\right\|^{2} \mathrm{~d} \tau \\
& \leq \frac{1}{\beta_{2}}\left\|f_{7}\right\|_{L_{g}}^{2}<\infty,
\end{aligned}
$$

since $f_{7} \in L_{g}$. Therefore,

$$
s \mapsto \int_{0}^{s} f_{7}(\tau) \mathrm{d} \tau \in L_{g} .
$$

Consequently, (2.32), (2.33) and (2.34) prove that $\eta \in L_{g}$.

Third, from (2.32), we see that the second, fourth and sixth equations in (2.29) are reduced to

$$
\left\{\begin{array}{l}
k\left(\varphi_{x}+\psi+w\right)_{x}=\rho_{1} f_{2}, \\
\left(b-g_{0}\right) \psi_{x x}-k\left(\varphi_{x}+\psi+w\right)=\rho_{2} f_{4}-\int_{0}^{\infty} g(s)\left(s f_{3}-\int_{0}^{s} f_{7}(\tau) \mathrm{d} \tau\right)_{x x} \mathrm{~d} s, \\
k_{0} w_{x x}-k\left(\varphi_{x}+\psi+w\right)-\delta w=\rho_{3} f_{6} .
\end{array}\right.
$$

Finally, (2.29) has a solution $U \in D(\mathcal{A})$ if and only if (2.35) has a solution

$$
(\varphi, \psi, w) \in H_{1} \times H_{0} \times H_{0}
$$

satisfying

$$
\varphi_{x}(0)=\psi_{x}(1)=w_{x}(1)=0
$$

and the regularity $\mathcal{A} U \in \mathcal{H}$ required in $D(\mathcal{A})$ (in case (2.3)), that is

$$
\varphi_{x x}, w_{x x} \in L^{2}(0,1) \quad \text { and } \quad\left(b-g_{0}\right) \psi_{x x}+\int_{0}^{\infty} g(s) \eta_{x x} \mathrm{~d} s \in L^{2}(0,1) .
$$

By considering the variational formulation of (2.35) in $H_{1} \times H_{0} \times H_{0}$ and using (2.20), the Lax-Milgram theorem and classical elliptic regularity arguments, it follows that (2.35) admits a unique solution $(\varphi, \psi, w)$ satisfying (2.36), (2.37) and (2.38). This proves that (2.29) has a unique solution $U \in D(\mathcal{A})$. By the resolvent identity, we have $\lambda I-\mathcal{A}$ is surjective, for any $\lambda>0$ (Liu \& Zheng, 1999), where $I$ denotes the identity operator. So, the Lumer-Phillips theorem implies that $\mathcal{A}$ is the infinitesimal generator of a linear $C_{0}$ semigroup of contractions on $\mathcal{H}$ and $D(\mathcal{A})$ is dense in $\mathcal{H}$. So, Theorem 2.1 holds (Pazy, 1983).

The proof of our stability results is based on the following frequency domain theorems: 
Theorem 2.2 (Huang, 1985 and Prüss, 1984) A $C_{0}$ semigroup of contractions on a Hilbert space $\mathcal{H}$ generated by an operator $\mathcal{A}$ is exponentially stable if and only if

$$
i \mathbb{R} \subset \rho(\mathcal{A}) \quad \text { and } \quad \sup _{\lambda \in \mathbb{R}}\left\|(i \lambda I-\mathcal{A})^{-1}\right\|_{\mathcal{L}(\mathcal{H})}<\infty .
$$

Theorem 2.3 (Liu \& Rao, 2005) If a bounded $C_{0}$ semigroup $e^{t \mathcal{A}}$ on a Hilbert space $\mathcal{H}$ generated by an operator $\mathcal{A}$ satisfies, for some $j \in \mathbb{N}^{*}$,

$$
i \mathbb{R} \subset \rho(\mathcal{A}) \quad \text { and } \quad \sup _{|\lambda| \geq 1} \frac{1}{\lambda^{j}}\left\|(i \lambda I-\mathcal{A})^{-1}\right\|_{\mathcal{L}(\mathcal{H})}<\infty
$$

then, for any $m \in \mathbb{N}^{*}$, there exists a positive constant $c_{m}$ such that

$$
\left\|e^{t \mathcal{A}} U_{0}\right\|_{\mathcal{H}} \leq c_{m}\left\|U_{0}\right\|_{D\left(\mathcal{A}^{m}\right)}\left(\frac{\ln t}{t}\right)^{\frac{m}{j}} \ln t, \quad U_{0} \in D\left(\mathcal{A}^{m}\right), t>0 .
$$

\section{Strong stability}

In this section, we prove our first stability result concerning the strong stability of our $C_{0}$ semigroup $e^{t \mathcal{A}}$ generated by the operator $\mathcal{A}$.

Theorem 3.1 Assume that (H) holds. Then, for any $U_{0} \in \mathcal{H}$, the solution $U$ of (2.12) in cases (2.3) and (2.4) satisfies

$$
\lim _{t \rightarrow \infty}\|U(t)\|_{\mathcal{H}}=0 .
$$

However, (3.1) holds in case (2.2) if moreover

$$
\left(\frac{\rho_{3} b}{\rho_{2}}-k_{0}\right)\left(\frac{\pi}{2}+m \pi\right)^{2} \neq \delta, \quad m \in \mathbb{N} .
$$

Remark 2 Condition (3.2) holds if, for example,

$$
\frac{b}{\rho_{2}}=\frac{k_{0}}{\rho_{3}}
$$

which means that the speeds of wave propagations of the last two equations in (2.1) are equal. The property (3.3) holds in fact from the physical point of view.

Proof. (Theorem 3.1) A $C_{0}$ semigroup of contractions $e^{t \mathcal{A}}$ generated by an operator $\mathcal{A}$ on a Hilbert space $\mathcal{H}$ is strongly stable if $\mathcal{A}$ has no imaginary eigenvalues and $\sigma(\mathcal{A}) \cap i \mathbb{R}$ is countable, where $\sigma(\mathcal{A})$ is the spectrum set of $\mathcal{A}$ (Arendt \& Batty, 1988). According to the fact that $0 \in \rho(\mathcal{A})$ (proved in Section 2) and since $D(\mathcal{A})$ has a compact embedding into $\mathcal{H}$, the linear bounded operator $\mathcal{A}^{-1}$ is a bijection between $\mathcal{H}$ and $D(\mathcal{A})$, and $\mathcal{A}^{-1}$ is a compact operator, which implies that $\sigma(\mathcal{A})$ is discrete and has only eigenvalues. Consequently, to get (3.1), we only have to prove that there is no imaginary 
eigenvalues for $\mathcal{A}$, that is

$$
\operatorname{ker}(i \lambda I-\mathcal{A})=\{0\}
$$

In Section 2, we have proved (3.4) for $\lambda=0$. So let $\lambda \in \mathbb{R}^{*}$ and

$$
U=(\varphi, \tilde{\varphi}, \psi, \tilde{\psi}, w, \tilde{w}, \eta) \in D(\mathcal{A})
$$

such that

$$
i \lambda U-\mathcal{A} U=0 .
$$

We have to prove that $U=0$. From (2.28), we have

$$
0=\operatorname{Re} i \lambda\|U\|_{\mathcal{H}}^{2}=\operatorname{Re}\langle i \lambda U, U\rangle_{\mathcal{H}}=\operatorname{Re}\langle\mathcal{A} U, U\rangle_{\mathcal{H}}=\frac{1}{2} \int_{0}^{\infty} g^{\prime}(s)\left\|\eta_{x}\right\|^{2} \mathrm{~d} s .
$$

Therefore, using the second inequality in (2.21),

$$
0 \leq\|\eta\|_{L_{g}}^{2}=\int_{0}^{\infty} g(s)\left\|\eta_{x}\right\|^{2} \mathrm{~d} s \leq \frac{-1}{\beta_{2}} \int_{0}^{\infty} g^{\prime}(s)\left\|\eta_{x}\right\|^{2} \mathrm{~d} s=0,
$$

so

$$
\eta=0
$$

By the last equation in (3.5), we find

$$
\tilde{f}=0,
$$

where $\tilde{f}$ is defined in (2.10). Now, we discuss the case of each system alone.

\subsection{Case (2.2)}

In virtue of (2.10), (2.13), (2.14), (3.7) and the first equation in (3.5), we have

$$
\varphi=\tilde{\varphi}=0
$$

Then (3.5), (3.6) and (3.8) lead to

$$
\left\{\begin{array}{l}
\tilde{\psi}=i \lambda \psi, \quad \tilde{w}=i \lambda w \\
(\psi+w)_{x}=0 \\
b \psi_{x x}-k(\psi+w)=-\rho_{2} \lambda^{2} \psi \\
k_{0} w_{x x}-k(\psi+w)-\delta w=-\rho_{3} \lambda^{2} w
\end{array}\right.
$$

The third equation in (3.9) implies that $\psi+w$ is a constant. Because $\psi+3 w$ vanishes at $x=0$, then we get

$$
\psi+w=0
$$


Combining (3.10) and the fourth equation in (3.9), we obtain

$$
\psi_{x x}+\frac{\rho_{2}}{b} \lambda^{2} \psi=0
$$

This implies that, for $c_{1}, c_{2} \in \mathbb{C}$,

$$
\psi(x)=c_{1} \cos \left(\sqrt{\frac{\rho_{2}}{b} \lambda^{2} x}\right)+c_{2} \sin \left(\sqrt{\frac{\rho_{2}}{b} \lambda^{2} x}\right) .
$$

The boundary condition $\psi(0)=0$ leads to $c_{1}=0$, and then

$$
\psi(x)=c_{2} \sin \left(\sqrt{\frac{\rho_{2}}{b} \lambda^{2} x}\right) .
$$

Assume by contradiction that $c_{2} \neq 0$. Because $\psi_{x}(1)=0$, we have

$$
\exists m \in \mathbb{N}: \sqrt{\frac{\rho_{2}}{b} \lambda^{2}}=\frac{\pi}{2}+m \pi .
$$

Therefore, combining (3.10) and (3.12) and using the last equation in (3.9), we see that

$$
\left(\frac{\rho_{2} k_{0}}{b}-\rho_{3}\right) \lambda^{2}+\delta=0 .
$$

So, combining (3.13) and (3.14), we get

$$
\left(\frac{\rho_{3} b}{\rho_{2}}-k_{0}\right)\left(\frac{\pi}{2}+m \pi\right)^{2}=\delta
$$

which is a contradiction with (3.2). Consequently, $c_{2}=0$ and hence

$$
\psi=0
$$

Using (3.10) and the first two equations in (3.9) we obtain

$$
w=\tilde{\psi}=\tilde{w}=0
$$

So, according to (3.6), (3.8), (3.15) and (3.16), we conclude that $U=0$.

\subsection{Case (2.3)}

From (2.10), (2.13), (2.15), (3.7) and the third equation in (3.5), we have

$$
\psi=\tilde{\psi}=0
$$


Then (3.5), (3.6) and (3.17) lead to

$$
\left\{\begin{array}{l}
\tilde{\varphi}=i \lambda \varphi, \quad \tilde{w}=i \lambda w \\
k\left(\varphi_{x}+w\right)_{x}=-\rho_{1} \lambda^{2} \varphi \\
\varphi_{x}+w=0 \\
k_{0} w_{x x}-k\left(\varphi_{x}+w\right)-\delta w=-\rho_{3} \lambda^{2} w
\end{array}\right.
$$

The third and fourth equations in (3.18) implies that

$$
\varphi=w=0,
$$

and then the first and second equations in (3.18) lead to

$$
\tilde{\varphi}=\tilde{w}=0 .
$$

The above properties (3.6), (3.17), (3.19) and (3.20) show that $U=0$.

\subsection{Case (2.4)}

As in the previous case, (2.10), (2.13), (2.16), (3.7) and the fifth equation in (3.5) give

$$
w=\tilde{w}=0 .
$$

Then (3.5), (3.6) and (3.21) lead to

$$
\left\{\begin{array}{l}
\tilde{\varphi}=i \lambda \varphi, \quad \tilde{\psi}=i \lambda \psi \\
k\left(\varphi_{x}+\psi\right)_{x}=-\rho_{1} \lambda^{2} \varphi, \\
b \psi_{x x}-k\left(\varphi_{x}+\psi\right)=-\rho_{2} \lambda^{2} \psi \\
\varphi_{x}+\psi=0 .
\end{array}\right.
$$

The third and fifth equations in (3.22) imply that

$$
\varphi=\psi=0
$$

hence, the first two equations in (3.22) lead to

$$
\tilde{\varphi}=\tilde{\psi}=0
$$

So (3.6), (3.21), (3.23) and (3.24) prove that $U=0$.

Finally, (3.4) holds and thus the proof of Theorem 3.1 is ended.

\section{Exponential stability}

In this section, we show that (2.12) in cases (2.3) and (2.4) is exponentially stable if the three speeds of wave propagations are equal, that is we have the following theorem: 
THEOREM 4.1 We assume that $(\mathbf{H})$ holds and

$$
\frac{k}{\rho_{1}}=\frac{b}{\rho_{2}}=\frac{k_{0}}{\rho_{3}} .
$$

Then there exist two positive constants $C_{0}$ and $C_{1}$ such that the $C_{0}$-semigroup $e^{t \mathcal{A}}$ on $\mathcal{H}$ in cases (2.3) and (2.4) satisfies the estimate

$$
\left\|e^{t \mathcal{A}}\right\|_{\mathcal{L}(\mathcal{H})} \leq C_{1} e^{-C_{0} t}, \quad t>0
$$

Proof. Using Theorem 2.2, we have to show (2.39). We have already proved in Section 3 that

$$
i \mathbb{R} \subset \rho(\mathcal{A})
$$

So we only need to prove that

$$
\sup _{|\lambda| \geq 1}\left\|(i \lambda I-\mathcal{A})^{-1}\right\|_{\mathcal{L}(\mathcal{H})}<\infty .
$$

Let us prove (4.4) by contradiction. Assume that (4.4) does not hold, then there exist two sequences $\left(U_{n}\right)_{n} \subset D(\mathcal{A})$ and $\left(\lambda_{n}\right)_{n} \subset \mathbb{R}$ satisfying

$$
\begin{aligned}
\left\|U_{n}\right\|_{\mathcal{H}} & =1, \quad n \in \mathbb{N}, \\
\lim _{n \rightarrow \infty}\left|\lambda_{n}\right| & =\infty
\end{aligned}
$$

and

$$
\lim _{n \rightarrow \infty}\left\|\left(i \lambda_{n} I-\mathcal{A}\right) U_{n}\right\|_{\mathcal{H}}=0
$$

Let $U_{n}=\left(\varphi_{n}, \tilde{\varphi}_{n}, \psi_{n}, \tilde{\psi}_{n}, w_{n}, \tilde{w}_{n}, \eta_{n}\right)$. Using (2.13)-(2.16), we notice that the limit (4.7) implies that

$$
\left\{\begin{array}{l}
i \lambda_{n} \varphi_{n}-\tilde{\varphi}_{n} \rightarrow 0 \text { in } H_{1}, \\
i \lambda_{n} \rho_{1} \tilde{\varphi}_{n}-k\left(\varphi_{n x}+\psi_{n}+w_{n}\right)_{x}+G_{1, n x x} \rightarrow 0 \text { in } L^{2}(0,1), \\
i \lambda_{n} \psi_{n}-\tilde{\psi}_{n} \rightarrow 0 \text { in } H_{0}, \\
i \lambda_{n} \rho_{2} \tilde{\psi}_{n}-b \psi_{n x x}+k\left(\varphi_{n x}+\psi_{n}+w_{n}\right)+G_{2, n x x} \rightarrow 0 \text { in } L^{2}(0,1), \\
i \lambda_{n} w_{n}-\tilde{w}_{n} \rightarrow 0 \text { in } H_{0}, \\
i \lambda_{n} \rho_{3} \tilde{w}_{n}-k_{0} w_{n x x}+k\left(\varphi_{n x}+\psi_{n}+w_{n}\right)+\delta w_{n}+G_{3, n x x} \rightarrow 0 \text { in } L^{2}(0,1), \\
i \lambda_{n} \eta_{n}+\eta_{n s}-\tilde{f}_{n} \rightarrow 0 \text { in } L_{g}
\end{array}\right.
$$


as when $n$ goes to infinity, where

$$
\left\{\begin{array}{l}
f_{n}=\varphi_{n}, \quad \tilde{f}_{n}=\tilde{\varphi}_{n}, \quad G_{2, n}=G_{3, n}=0 \quad \text { and } \quad G_{1, n}=g_{0} f_{n}-\int_{0}^{\infty} g \eta_{n} \mathrm{~d} s \quad \text { in case (2.2), } \\
f_{n}=\psi_{n}, \quad \tilde{f}_{n}=\tilde{\psi}_{n}, \quad G_{1, n}=G_{3, n}=0 \quad \text { and } \quad G_{2, n}=g_{0} f_{n}-\int_{0}^{\infty} g \eta_{n} \mathrm{~d} s \quad \text { in case (2.3), } \\
f_{n}=w_{n}, \quad \tilde{f}_{n}=\tilde{w}_{n}, \quad G_{1, n}=G_{2, n}=0 \quad \text { and } \quad G_{3, n}=g_{0} f_{n}-\int_{0}^{\infty} g \eta_{n} \mathrm{~d} s \quad \text { in case (2.4) }
\end{array}\right.
$$

and $\eta$ is defined in (2.7). We will prove that

$$
\left\|U_{n}\right\|_{\mathcal{H}} \longrightarrow 0
$$

which gives a contradiction with (4.5). Using (2.28), we get

$$
\operatorname{Re}\left\langle\left(i \lambda_{n} I-\mathcal{A}\right) U_{n}, U_{n}\right\rangle_{\mathcal{H}}=\operatorname{Re}\left(i \lambda_{n}\left\|U_{n}\right\|_{\mathcal{H}}^{2}-\left\langle\mathcal{A} U_{n}, U_{n}\right\rangle_{\mathcal{H}}\right)=\frac{-1}{2} \int_{0}^{\infty} g^{\prime}(s)\left\|\eta_{n x}\right\|^{2} \mathrm{~d} s
$$

So (4.5) and (4.7) imply that

$$
\int_{0}^{\infty} g^{\prime}(s)\left\|\eta_{n x}\right\|^{2} \mathrm{~d} s \longrightarrow 0 .
$$

But, using the second inequality in (2.21), we have

$$
0 \leq \int_{0}^{\infty} g(s)\left\|\eta_{n x}\right\|^{2} \mathrm{~d} s \leq \frac{-1}{\beta_{2}} \int_{0}^{\infty} g^{\prime}(s)\left\|\eta_{n x}\right\|^{2} \mathrm{~d} s
$$

then (4.11) and (4.12) give

$$
\int_{0}^{\infty} g(s)\left\|\eta_{n x}\right\|^{2} \mathrm{~d} s \longrightarrow 0
$$

hence

$$
\eta_{n} \longrightarrow 0 \text { in } L_{g} \text {. }
$$

Using (4.5) and (4.6), we get from the last limit in (4.8) that

$$
\left\langle\left(i \lambda_{n} \eta_{n}+\eta_{n s}-i \lambda_{n} f_{n}+i \lambda_{n} f_{n}-\tilde{f}_{n}\right), i f_{n}\right\rangle_{L_{g}} \longrightarrow 0
$$

On the other hand, using $(4.8)_{3}$ in case (2.3), and (4.8) $)_{5}$ in case (2.4), we get (notice that the case (2.2) is not concerned by Theorem 4.1)

$$
\left\langle\left(i \lambda_{n} f_{n}-\tilde{f}_{n}\right), i f_{n}\right\rangle_{L_{g}} \longrightarrow 0
$$


So (4.6), (4.14) and (4.15) imply that

$$
\left\langle\eta_{n}, f_{n}\right\rangle_{L_{g}}-\frac{i}{\lambda_{n}}\left\langle\eta_{n s}, f_{n}\right\rangle_{L_{g}}-\left\langle f_{n}, f_{n}\right\rangle_{L_{g}} \longrightarrow 0
$$

We see that

$$
-\left\langle f_{n}, f_{n}\right\rangle_{L_{g}}=-g_{0}\left\|f_{n x}\right\|^{2}
$$

On the other hand, (4.5) and (4.13) imply that

$$
\left\langle\eta_{n}, f_{n}\right\rangle_{L_{g}} \longrightarrow 0
$$

Moreover, integrating by part with respect to $s$, applying Cauchy-Schwartz inequality and using the first inequality in (2.21) and the fact that

$$
\eta_{n x}(x, 0)=0 \quad \text { and } \quad \lim _{s \rightarrow \infty} g(s)=0
$$

we get

$$
\begin{aligned}
\left|\left\langle\eta_{n s}, f_{n}\right\rangle_{L_{g}}\right| & =\left|\left\langle f_{n x}, \int_{0}^{\infty}\left(-g^{\prime}(s)\right) \eta_{n x} \mathrm{~d} s\right\rangle\right| \\
& \leq\left\|f_{n x}\right\| \int_{0}^{\infty}\left(-g^{\prime}(s)\right)\left\|\eta_{n x}\right\| \mathrm{d} s \\
& \leq \sqrt{g(0)}\left\|f_{n x}\right\|\left(\int_{0}^{\infty}\left(-g^{\prime}(s)\right)\left\|\eta_{n x}\right\|^{2} \mathrm{~d} s\right)^{\frac{1}{2}} \\
& \leq \sqrt{\beta_{1} g(0)}\left\|f_{n x}\right\|\left\|\eta_{n}\right\|_{L_{g}},
\end{aligned}
$$

and then, according to (4.5) and (4.13),

$$
\left\langle\eta_{n s}, f_{n}\right\rangle_{L_{g}} \longrightarrow 0
$$

Consequently, (4.16), (4.17), (4.18), (4.21) and since $g_{0}>0$ (hypothesis (H)) lead to

$$
f_{n x} \longrightarrow 0 \text { in } L^{2}(0,1) \text {. }
$$

Moreover, because $f_{n}$ vanishes at $x=0$ (notice that $f_{n}=\psi_{n}$ in case (2.3), and $f_{n}=w_{n}$ in case (2.4)), then

$$
f_{n} \longrightarrow 0 \text { in } L^{2}(0,1)
$$

Multiplying $(4.8)_{1},(4.8)_{3}$ and (4.8) $)_{5}$ by $\frac{1}{\lambda_{n}}$ and using (4.6) and (4.22), we obtain

$$
\frac{1}{\lambda_{n}} \tilde{f}_{n x} \longrightarrow 0 \text { in } L^{2}(0,1) \text {. }
$$


Similarily, multiplying $(4.8)_{1},(4.8)_{3}$ and (4.8) $)_{5}$ by $\frac{1}{\lambda_{n}}$ and using (4.5) and (4.6), we obtain

$$
\left\{\begin{array}{l}
\varphi_{n} \longrightarrow 0 \text { in } L^{2}(0,1), \\
\psi_{n} \longrightarrow 0 \text { in } L^{2}(0,1), \\
w_{n} \longrightarrow 0 \text { in } L^{2}(0,1) .
\end{array}\right.
$$

Moreover, using (4.5), we deduce from $(4.8)_{1},(4.8)_{3}$ and $(4.8)_{5}$ that

$$
\left(\lambda_{n} \varphi_{n}\right)_{n},\left(\lambda_{n} \psi_{n}\right)_{n} \text { and }\left(\lambda_{n} w_{n}\right)_{n} \text { are bounded in } L^{2}(0,1)
$$

Now, we distinguish the cases (2.3) and (2.4).

\subsection{Case (2.3)}

We have in this case $f_{n}=\psi_{n}, \tilde{f}_{n}=\tilde{\psi}_{n}$ and $G_{1, n}=G_{3, n}=0$. Taking the inner product of $(4.8)_{4}$ with $\frac{i \tilde{\psi}_{n}}{\lambda_{n}}$ in $L^{2}(0,1)$, integrating by parts and using the boundary conditions, we see that

$$
\rho_{2}\left\|\tilde{\psi}_{n}\right\|{ }^{2}+\left\langle\left(b \psi_{n x}-G_{2, n x}\right), \frac{i \tilde{\psi}_{n x}}{\lambda_{n}}\right\rangle+k\left\langle\left(\varphi_{n x}+\psi_{n}+w_{n}\right), \frac{i \tilde{\psi}_{n}}{\lambda_{n}}\right\rangle \rightarrow 0 .
$$

Using (4.5), (4.6) and (4.24), we obtain

$$
\tilde{\psi}_{n} \longrightarrow 0 \text { in } L^{2}(0,1)
$$

and with $(4.8)_{3}$, we find

$$
\lambda_{n} \psi_{n} \longrightarrow 0 \text { in } L^{2}(0,1)
$$

Taking the inner product of $\left(\varphi_{n x}+\psi_{n}+w_{n}\right)$ with $i \lambda_{n} \tilde{\psi}_{n}$ in $L^{2}(0,1)$, integrating by parts and using the boundary conditions, we have

$$
\begin{aligned}
\left\langle\left(\varphi_{n x}+\psi_{n}+w_{n}\right), i \lambda_{n} \tilde{\psi}_{n}\right\rangle= & -\left\langle i \lambda_{n} \varphi_{n x}, \tilde{\psi}_{n}\right\rangle-\left\langle i \lambda_{n} \psi_{n}, \tilde{\psi}_{n}\right\rangle-\left\langle i \lambda_{n} w_{n}, \tilde{\psi}_{n}\right\rangle \\
= & \left\langle\left(i \lambda_{n} \varphi_{n}-\tilde{\varphi}_{n}\right), \tilde{\psi}_{n x}\right\rangle+\left\langle\tilde{\varphi}_{n}, \tilde{\psi}_{n x}\right\rangle-\left\langle\left(i \lambda_{n} \psi_{n}-\tilde{\psi}_{n}\right), \tilde{\psi}_{n}\right\rangle \\
& -\left\|\tilde{\psi}_{n}\right\|^{2}-\left\langle\tilde{w}_{n}, \tilde{\psi}_{n}\right\rangle-\left\langle\left(i \lambda_{n} w_{n}-\tilde{w}_{n}\right), \tilde{\psi}_{n}\right\rangle \\
= & -\left\langle\left(i \lambda_{n} \varphi_{n x}-\tilde{\varphi}_{n x}\right), \tilde{\psi}_{n}\right\rangle+\left\langle\tilde{\varphi}_{n}, \tilde{\psi}_{n x}\right\rangle-\left\langle\left(i \lambda_{n} \psi_{n}-\tilde{\psi}_{n}\right), \tilde{\psi}_{n}\right\rangle \\
& -\left\|\tilde{\psi}_{n}\right\|^{2}-\left\langle\tilde{w}_{n}, \tilde{\psi}_{n}\right\rangle-\left\langle\left(i \lambda_{n} w_{n}-\tilde{w}_{n}\right), \tilde{\psi}_{n}\right\rangle .
\end{aligned}
$$

Then, by using (4.5), (4.8) $1,(4.8)_{3},(4.8)_{5}$ and (4.27), we deduce that

$$
\left\langle\left(\varphi_{n x}+\psi_{n}+w_{n}\right), i \lambda_{n} \tilde{\psi}_{n}\right\rangle-\left\langle\tilde{\varphi}_{n}, \tilde{\psi}_{n x}\right\rangle \longrightarrow 0 .
$$


Taking the inner product of $\tilde{\varphi}_{n}$ with $\tilde{\psi}_{n x}$ in $L^{2}(0,1)$, we arrive at

$$
\begin{aligned}
\left\langle\tilde{\varphi}_{n}, \tilde{\psi}_{n x}\right\rangle & =\left\langle\tilde{\varphi}_{n},\left(\tilde{\psi}_{n x}-i \lambda_{n} \psi_{n x}+i \lambda_{n} \psi_{n x}-\frac{i \lambda_{n}}{b} G_{2, n x}+\frac{i \lambda_{n}}{b} G_{2, n x}\right)\right\rangle \\
& =-\left\langle\tilde{\varphi}_{n},\left(i \lambda_{n} \psi_{n x}-\tilde{\psi}_{n x}\right)\right\rangle+\left\langle\tilde{\varphi}_{n}, \frac{i \lambda_{n}}{b}\left(b \psi_{n x}-G_{2, n x}\right)\right\rangle+\left\langle\tilde{\varphi}_{n}, \frac{i \lambda_{n}}{b} G_{2, n x}\right\rangle,
\end{aligned}
$$

then, by (4.5) and (4.8) 3 , we have

$$
\left\langle\tilde{\varphi}_{n}, \frac{i \lambda_{n}}{b}\left(b \psi_{n x}-G_{2, n x}\right)\right\rangle+\left\langle\tilde{\varphi}_{n}, \frac{i \lambda_{n}}{b} G_{2, n x}\right\rangle-\left\langle\tilde{\varphi}_{n}, \tilde{\psi}_{n x}\right\rangle \longrightarrow 0
$$

Taking the inner product of $(4.8)_{2}$ with $\frac{1}{b}\left(b \psi_{n x}-G_{2, n x}\right)$ in $L^{2}(0,1)$, integrating by parts and using the boundary conditions, we find

$$
\left\langle i \lambda_{n} \rho_{1} \tilde{\varphi}_{n}, \frac{1}{b}\left(b \psi_{n x}-G_{2, n x}\right)\right\rangle+k\left\langle\left(\varphi_{n x}+\psi_{n}+w_{n}\right), \frac{1}{b}\left(b \psi_{n x x}-G_{2, n x x}\right)\right\rangle \longrightarrow 0,
$$

which implies that

$$
\begin{aligned}
& -\rho_{1}\left\langle\tilde{\varphi}_{n}, \frac{i \lambda_{n}}{b}\left(b \psi_{n x}-G_{2, n x}\right)\right\rangle \\
& +k\left\langle\left(\varphi_{n x}+\psi_{n}+w_{n}\right), \frac{-1}{b}\left(i \lambda_{n} \rho_{2} \tilde{\psi}_{n}-b \psi_{n x x}+k\left(\varphi_{n x}+\psi_{n}+w_{n}\right)+G_{2, n x x}\right)\right\rangle \\
& +\frac{\rho_{2} k}{b}\left\langle\lambda_{n}\left(\psi_{n}+w_{n}\right), i \tilde{\psi}_{n}\right\rangle+\frac{\rho_{2} k}{b}\left\langle\varphi_{n x}, i \lambda_{n} \tilde{\psi}_{n}\right\rangle+\frac{k^{2}}{b}\left\|\varphi_{n x}+\psi_{n}+w_{n}\right\|^{2} \longrightarrow 0 .
\end{aligned}
$$

Using (4.5), (4.8) $)_{4},(4.26)$ and (4.27), we find

$$
-\rho_{1}\left\langle\tilde{\varphi}_{n}, \frac{i \lambda_{n}}{b}\left(b \psi_{n x}-G_{2, n x}\right)\right\rangle+\frac{\rho_{2} k}{b}\left\langle\varphi_{n x}, i \lambda_{n} \tilde{\psi}_{n}\right\rangle+\frac{k^{2}}{b}\left\|\varphi_{n x}+\psi_{n}+w_{n}\right\|^{2} \longrightarrow 0
$$

Using the definition of $G_{2, n}$ in (4.9) in case (2.3), integrating by parts (with respect to $s$ ) and using the boundary conditions, we get

$$
\begin{aligned}
\left\langle\tilde{\varphi}_{n}, \frac{i \lambda_{n}}{b} G_{2, n x}\right\rangle= & \left\langle\tilde{\varphi}_{n}, \frac{1}{b}\left(i g_{0} \lambda_{n} \psi_{n x}-\int_{0}^{\infty} g\left(i \lambda_{n} \eta_{n x}+\eta_{n x s}-\tilde{\psi}_{n x}-\eta_{n x s}+\tilde{\psi}_{n x}\right) \mathrm{d} s\right)\right\rangle \\
= & -\left\langle\tilde{\varphi}_{n}, \frac{1}{b}\left(\int_{0}^{\infty} g\left(i \lambda_{n} \eta_{n x}+\eta_{n x s}-\tilde{\psi}_{n x}\right) \mathrm{d} s\right)\right\rangle \\
& +\left\langle\tilde{\varphi}_{n}, \frac{g_{0}}{b}\left(i \lambda_{n} \psi_{n x}-\tilde{\psi}_{n x}\right)\right\rangle+\left\langle\tilde{\varphi}_{n}, \frac{-1}{b} \int_{0}^{\infty} g^{\prime} \eta_{n x} \mathrm{~d} s\right\rangle
\end{aligned}
$$


therefore, from $(4.5),(4.8)_{3},(4.8)_{7}$ and (4.11), we obtain

$$
\left\langle\tilde{\varphi}_{n}, \frac{i \lambda_{n}}{b} G_{2, n x}\right\rangle \rightarrow 0
$$

Then, multiplying (4.29) by $\frac{-\rho_{2} k}{b}$, and (4.30) by $\rho_{1}$, adding the obtained limits and (4.31) and using (4.32), we find

$$
\left(\frac{\rho_{2} k}{b}-\rho_{1}\right)\left\langle\tilde{\varphi}_{n}, \tilde{\psi}_{n x}\right\rangle+\frac{k^{2}}{b}\left\|\left(\varphi_{n x}+\psi_{n}+w_{n}\right)\right\|^{2}+\frac{i \rho_{2} k}{b}\left\langle\lambda_{n} \psi_{n}+\lambda_{n} w_{n}, \tilde{\psi}_{n}\right\rangle \longrightarrow 0 .
$$

So, because $\frac{\rho_{2} k}{b}-\rho_{1}=0$ (according to (4.1)), we get from (4.25), (4.26), (4.27) and (4.33) that

$$
\varphi_{n x} \longrightarrow 0 \text { in } L^{2}(0,1)
$$

and using (4.6) and (4.8) 1 , we get

$$
\frac{\tilde{\varphi}_{n x}}{\lambda_{n}} \longrightarrow 0 \text { in } L^{2}(0,1)
$$

Multiplying (4.8) $)_{2}$ by $\frac{i \tilde{\varphi}_{n}}{\lambda_{n}}$, integrating by parts and using the boundary conditions, we arrive at

$$
\rho_{1}\left\|\tilde{\varphi}_{n}\right\|^{2}+k\left\langle\left(\varphi_{n x}+\psi_{n}+w_{n}\right), \frac{i \tilde{\varphi}_{n x}}{\lambda_{n}}\right\rangle \longrightarrow 0,
$$

then, using (4.5) and (4.35), we have

$$
\tilde{\varphi}_{n} \longrightarrow 0 \text { in } L^{2}(0,1)
$$

and therefore, using $(4.8)_{1}$,

$$
\lambda_{n} \varphi_{n} \longrightarrow 0 \text { in } L^{2}(0,1) .
$$

Taking the inner product of $(4.8)_{6}$ with $\left(\varphi_{n x}+\psi_{n}+w_{n}\right)$ in $L^{2}(0,1)$, integrating by parts and using the boundary conditions, we get

$$
\begin{aligned}
\left\langle i \lambda_{n} \rho_{3} \tilde{w}_{n}, \varphi_{n x}\right\rangle+\left\langle i \lambda_{n} \rho_{3} \tilde{w}_{n}, \psi_{n}\right\rangle & +\left\langle i \lambda_{n} \rho_{3} \tilde{w}_{n}, w_{n}\right\rangle \\
& +k_{0}\left\langle w_{n x},\left(\varphi_{n x}+\psi_{n}+l w_{n}\right)_{x}\right\rangle+k\left\|\left(\varphi_{n x}+\psi_{n}+l w_{n}\right)\right\|^{2} \longrightarrow 0,
\end{aligned}
$$

then

$$
\begin{aligned}
& -\lambda_{n} \rho_{3}\left\langle\tilde{w}_{n}, i \varphi_{n x}\right\rangle-\rho_{3}\left\langle\tilde{w}_{n},\left(i \lambda_{n} \psi_{n}-\tilde{\psi}_{n}\right)\right\rangle-\rho_{3}\left\langle\tilde{w}_{n}, \tilde{\psi}_{n}\right\rangle-\rho_{3}\left\langle\tilde{w}_{n},\left(i \lambda_{n} w_{n}-\tilde{w}_{n}\right)\right\rangle-\rho_{3}\left\|\tilde{w}_{n}\right\|^{2} \\
& -\frac{k_{0}}{k}\left\langle w_{n x},\left[i \lambda_{n} \rho_{1} \tilde{\varphi}_{n}-k\left(\varphi_{n x}+\psi_{n}+w_{n}\right)_{x}\right]\right\rangle+\frac{k_{0}}{k}\left\langle w_{n x}, i \lambda_{n} \rho_{1} \tilde{\varphi}_{n}\right\rangle+k\left\|\varphi_{n x}+\psi_{n}+l w_{n}\right\|^{2} \longrightarrow 0,
\end{aligned}
$$


using (4.5), (4.8) $2,(4.8)_{3},(4.8)_{5},(4.25),(4.27)$ and (4.34), we get

$$
-\rho_{3} \lambda_{n}\left\langle\tilde{w}_{n}, i \varphi_{n x}\right\rangle-\rho_{3}\left\|\tilde{w}_{n}\right\|^{2}+\frac{\rho_{1} k_{0}}{k} \lambda_{n}\left\langle w_{n x}, i \tilde{\varphi}_{n}\right\rangle \longrightarrow 0
$$

Integrating by parts and using the boundary conditions, we obtain

$$
\begin{aligned}
\lambda_{n}\left\langle w_{n x}, i \tilde{\varphi}_{n}\right\rangle & =-\left\langle\left(i \lambda_{n} w_{n x}-\tilde{w}_{n x}\right), \tilde{\varphi}_{n}\right\rangle-\left\langle\tilde{w}_{n x}, \tilde{\varphi}_{n}\right\rangle=-\left\langle\left(i \lambda_{n} w_{n x}-\tilde{w}_{n x}\right), \tilde{\varphi}_{n}\right\rangle+\left\langle\tilde{w}_{n}, \tilde{\varphi}_{n x}\right\rangle \\
& =-\left\langle\left(i \lambda_{n} w_{n x}-\tilde{w}_{n x}\right), \tilde{\varphi}_{n}\right\rangle-\left\langle\tilde{w}_{n},\left(i \lambda_{n} \varphi_{n x}-\tilde{\varphi}_{n x}\right)\right\rangle+\left\langle\tilde{w}_{n}, i \lambda_{n} \varphi_{n x}\right\rangle .
\end{aligned}
$$

Therefore, from (4.5), (4.8) 1 and (4.8) $)_{5}$, we see that

$$
\lambda_{n}\left\langle w_{n x}, i \tilde{\varphi}_{n}\right\rangle-\lambda_{n}\left\langle\tilde{w}_{n}, i \varphi_{n x}\right\rangle \longrightarrow 0
$$

so, multiplying (4.40) by $-\rho_{3}$ and inserting the obtained limit into (4.39), we obtain

$$
\frac{\lambda_{n}}{k}\left(k_{0} \rho_{1}-k \rho_{3}\right)\left\langle w_{n x}, i \tilde{\varphi}_{n}\right\rangle-\rho_{3}\left\|\tilde{w}_{n}\right\|^{2} \longrightarrow 0 .
$$

Thus, because $k_{0} \rho_{1}-k \rho_{3}=0$ (condition (4.1)), we get from (4.41) that

$$
\tilde{w}_{n} \longrightarrow 0 \text { in } L^{2}(0,1)
$$

and by $(4.8)_{5}$ and (4.42), we deduce that

$$
\lambda_{n} w_{n} \longrightarrow 0 \text { in } L^{2}(0,1)
$$

Taking the inner product of $(4.8)_{6}$ with $w_{n}$ in $L^{2}(0,1)$, integrating by parts and using the boundary conditions, we get

$$
-\rho_{3}\left\langle\tilde{w}_{n}, i \lambda_{n} w_{n}\right\rangle+k_{0}\left\|w_{n x}\right\|^{2}+\left\langle k\left(\varphi_{n x}+\psi_{n}+w_{n}\right)+\delta w_{n}, w_{n}\right\rangle \longrightarrow 0,
$$

and by using (4.5), (4.25) and (4.43), we conclude from (4.44) that

$$
w_{n x} \longrightarrow 0 \text { in } L^{2}(0,1)
$$

A combination of (4.13), (4.22), (4.25), (4.27), (4.34), (4.37), (4.42) and (4.45) lead to (4.10), which is a contradiction with (4.5). Hence, (4.4) is valid. Consequently, (2.12) in case (2.3) is exponentially stable, that is (4.2) holds. 


\subsection{Case (2.4)}

We have in this case $f_{n}=w_{n}, \tilde{f}_{n}=\tilde{w}_{n}$ and $G_{1, n}=G_{2, n}=0$. The proof is very similar to the one given in Section 4.1 for the case (2.3).

Taking the inner product of $(4.8)_{6}$ with $\frac{i \tilde{w}_{n}}{\lambda_{n}}$ in $L^{2}(0,1)$, integrating by parts and using the boundary conditions, (4.5), (4.6) and (4.24), we obtain

$$
\tilde{w}_{n} \longrightarrow 0 \text { in } L^{2}(0,1)
$$

and with $(4.8)_{5}$, we find

$$
\lambda_{n} w_{n} \longrightarrow 0 \text { in } L^{2}(0,1)
$$

Taking the inner product of $\left(\varphi_{n x}+\psi_{n}+w_{n}\right)$ with $i \lambda_{n} \tilde{w}_{n}$ in $L^{2}(0,1)$, integrating by parts and using the boundary conditions, $(4.5),(4.8)_{1},(4.8)_{3},(4.8)_{5}$ and (4.46), we deduce that (as for (4.29))

$$
\left\langle\left(\varphi_{n x}+\psi_{n}+w_{n}\right), i \lambda_{n} \tilde{w}_{n}\right\rangle-\left\langle\tilde{\varphi}_{n}, \tilde{w}_{n x}\right\rangle \longrightarrow 0
$$

Taking the inner product of $\tilde{\varphi}_{n}$ with $\tilde{w}_{n x}$ in $L^{2}(0,1)$, and exploiting (4.5), (4.8) 5 and (4.25), we have (as for (4.30))

$$
\left\langle\tilde{\varphi}_{n}, \frac{i \lambda_{n}}{k_{0}}\left(k_{0} w_{n x}-G_{3, n x}\right)\right\rangle+\left\langle\tilde{\varphi}_{n}, \frac{i \lambda_{n}}{k_{0}} G_{3, n x}\right\rangle-\left\langle\tilde{\varphi}_{n}, \tilde{w}_{n x}\right\rangle \rightarrow 0 .
$$

Taking the inner product of $(4.8)_{2}$ with $\frac{1}{k_{0}}\left(k_{0} \psi_{n x}-G_{3, n x}\right)$ in $L^{2}(0,1)$, integrating by parts and using the boundary conditions, (4.5), (4.8) 6 , (4.25), (4.26) and (4.46), we find (as for (4.31))

$$
-\rho_{1}\left\langle\tilde{\varphi}_{n}, \frac{i \lambda_{n}}{k_{0}}\left(k_{0} w_{n x}-G_{3, n x}\right)\right\rangle+\frac{k^{2}}{k_{0}}\left\|\varphi_{n x}+\psi_{n}+w_{n}\right\|^{2}+\frac{\rho_{3} k}{k_{0}}\left\langle\varphi_{n x}, i \lambda_{n} \tilde{w}_{n}\right\rangle \longrightarrow 0 .
$$

Using the definition of $G_{3, n}$ in (4.9) in case (2.4), integrating by parts (with respect to $s$ ) and using the boundary conditions, (4.5), (4.8) 7 and (4.11), we obtain (as for (4.32))

$$
\left\langle\tilde{\varphi}_{n}, \frac{i \lambda_{n}}{k_{0}} G_{3, n x}\right\rangle \longrightarrow 0
$$

Then, multiplying (4.48) by $\frac{-\rho_{3} k}{k_{0}}$, and (4.49) by $\rho_{1}$, adding the obtained limits and (4.50) and using (4.51), we find (as for (4.33))

$$
\left(\frac{\rho_{3} k}{k_{0}}-\rho_{1}\right)\left\langle\tilde{\varphi}_{n}, \tilde{w}_{n x}\right\rangle+\frac{k^{2}}{k_{0}}\left\|\left(\varphi_{n x}+\psi_{n}+w_{n}\right)\right\|^{2}+\frac{i \rho_{3} k}{k_{0}}\left\langle\lambda_{n} \psi_{n}+\lambda_{n} w_{n}, \tilde{w}_{n}\right\rangle \longrightarrow 0 .
$$

So, because $\frac{\rho_{3} k}{k_{0}}-\rho_{1}=0$ (according to (4.1)), we get from (4.25), (4.26), (4.46) and (4.52) that (4.34) holds, and so we get (4.35).

Multiplying (4.8) $)_{2}$ by $\frac{i \tilde{\varphi}_{n}}{\lambda_{n}}$, integrating by parts and using the boundary conditions, (4.5) and (4.35), we arrive at (4.37), and therefore, using $(4.8)_{1},(4.38)$ holds. 
Taking the inner product of $(4.8)_{4}$ with $\left(\varphi_{n x}+\psi_{n}+w_{n}\right)$ in $L^{2}(0,1)$, integrating by parts, using the boundary conditions, $(4.5),(4.8)_{2},(4.8)_{3},(4.8)_{5},(4.25),(4.34)$ and $(4.46)$, we get

$$
-\rho_{2} \lambda_{n}\left\langle\tilde{\psi}_{n}, i \varphi_{n x}\right\rangle-\rho_{2}\left\|\tilde{\psi}_{n}\right\|^{2}+\frac{\rho_{1} b}{k} \lambda_{n}\left\langle\psi_{n x}, i \tilde{\varphi}_{n}\right\rangle \longrightarrow 0
$$

Integrating by parts and using the boundary conditions, (4.5), (4.8) $)_{1}$ and $(4.8)_{3}$, we see that (as for (4.40))

$$
\lambda_{n}\left\langle\psi_{n x}, i \tilde{\varphi}_{n}\right\rangle-\lambda_{n}\left\langle\tilde{\psi}_{n}, i \varphi_{n x}\right\rangle \longrightarrow 0
$$

so, multiplying (4.54) by $-\rho_{2}$ and inserting the obtained limit into (4.53), we obtain

$$
\frac{\lambda_{n}}{k}\left(b \rho_{1}-k \rho_{2}\right)\left\langle\psi_{n x}, i \tilde{\varphi}_{n}\right\rangle-\rho_{2}\left\|\tilde{\psi}_{n}\right\|^{2} \longrightarrow 0 .
$$

Thus, because $b \rho_{1}-k \rho_{2}=0$ (in virtue of condition (4.1)), we get from (4.55) that (4.27) holds, and so (4.28) is valid.

Taking the inner product of $(4.8)_{4}$ with $\psi_{n}$ in $L^{2}(0,1)$, integrating by parts and using the boundary conditions, we find (as for (4.44))

$$
-\rho_{2}\left\langle\tilde{\psi}_{n}, i \lambda_{n} \psi_{n}\right\rangle+b\left\|\psi_{n x}\right\|^{2}+k\left\langle\left(\varphi_{n x}+\psi_{n}+w_{n}\right), \psi_{n}\right\rangle \rightarrow 0
$$

therefore, using (4.5), (4.25) and (4.28), we get

$$
\psi_{n x} \longrightarrow 0 \text { in } L^{2}(0,1)
$$

A combination of (4.13), (4.22), (4.25), (4.27), (4.34), (4.37), (4.46) and (4.56) lead to (4.10), which is a contradiction with (4.5). Hence, (4.4) is valid. Consequently, (2.12) in case (2.4) is exponentially stable. The proof of Theorem 4.1 is now completed.

\section{Polynomial stability}

We prove in this section that the infinite memory is strong enough to stabilize (2.12) at least polynomially even if (4.1) does not hold. Our result is stated as follows:

Theorem 5.1 We assume that $(\mathbf{H})$ holds. Then, for any $m \in \mathbb{N}^{*}$, there exists a constant $c_{m}>0$ such that

$$
\forall U_{0} \in D\left(\mathcal{A}^{m}\right), \forall t>0,\|U(t)\|_{\mathcal{H}} \leq c_{m}\left\|U_{0}\right\|_{D\left(\mathcal{A}^{m}\right)}\left(\frac{\ln t}{t}\right)^{\frac{m}{j}} \ln t
$$

in cases (2.3) and (2.4) with $j=10$. Moreover, (5.1) holds in case (2.2) with $j=4$ if in addition (3.2) is satisfied.

REMARK 3 The estimate (5.1) implies that

$$
\forall m \in \mathbb{N}^{*}, \forall U_{0} \in D\left(\mathcal{A}^{m}\right), \forall \epsilon>0, \exists C_{m, \epsilon, U_{0}}>0:\|U(t)\|_{\mathcal{H}} \leq C_{m, \epsilon, U_{0}} t^{\frac{-m}{j}+\epsilon}, \quad t>0
$$


with $j=10$ in cases (2.3) and (2.4), and $j=4$ in case (2.2). So, for classical solutions $(m=1)$, the decay rate of $t \mapsto\|U(t)\|_{\mathcal{H}}$ is arbitrarily close to $t^{\frac{-1}{j}}$.

Proof. (Theorem 5.1) Using Theorem 2.3, we need to show (4.3) and

$$
\sup _{|\lambda| \geq 1} \frac{1}{\lambda^{j}}\left\|(i \lambda I-\mathcal{A})^{-1}\right\| \mathcal{L}(\mathcal{H})<\infty .
$$

We have already proved (4.3) in Section 3. Now, we establish (5.2) by contradiction. Assume that (5.2) is false, then there exist sequences $\left(U_{n}\right)_{n} \subset D(\mathcal{A})$ and $\left(\lambda_{n}\right)_{n} \subset \mathbb{R}$ satisfying (4.5), (4.6) and

$$
\lim _{n \rightarrow \infty} \lambda_{n}^{j}\left\|\left(i \lambda_{n} I-\mathcal{A}\right) U_{n}\right\|_{\mathcal{H}}=0
$$

Let $U_{n}=\left(\varphi_{n}, \tilde{\varphi}_{n}, \psi_{n}, \tilde{\psi}_{n}, w_{n}, \tilde{w}_{n}, \eta_{n}\right)$. According to (2.13)-(2.16), the limit (5.3) implies that

$$
\left\{\begin{array}{l}
\lambda_{n}^{j}\left[i \lambda_{n} \varphi_{n}-\tilde{\varphi}_{n}\right] \rightarrow 0 \text { in } H_{1}, \\
\lambda_{n}^{j}\left[i \lambda_{n} \rho_{1} \tilde{\varphi}_{n}-k\left(\varphi_{n x}+\psi_{n}+w_{n}\right)_{x}+G_{1, n x x}\right] \rightarrow 0 \text { in } L^{2}(0,1), \\
\lambda_{n}^{j}\left[i \lambda_{n} \psi_{n}-\tilde{\psi}_{n}\right] \rightarrow 0 \text { in } H_{0}, \\
\lambda_{n}^{j}\left[i \lambda_{n} \rho_{2} \tilde{\psi}_{n}-b \psi_{n x x}+k\left(\varphi_{n x}+\psi_{n}+w_{n}\right)+G_{2, n x x}\right] \rightarrow 0 \text { in } L^{2}(0,1), \\
\lambda_{n}^{j}\left[i \lambda_{n} w_{n}-\tilde{w}_{n}\right] \rightarrow 0 \text { in } H_{0}, \\
\lambda_{n}^{j}\left[i \lambda_{n} \rho_{3} \tilde{w}_{n}-k_{0} w_{n x x}+k\left(\varphi_{n x}+\psi_{n}+w_{n}\right)+\delta w_{n}+G_{3, n x x}\right] \rightarrow 0 \text { in } L^{2}(0,1), \\
\lambda_{n}^{j}\left[i \lambda_{n} \eta_{n}+\eta_{n s}-\tilde{f}_{n}\right] \rightarrow 0 \text { in } L_{g},
\end{array}\right.
$$

where $f_{n}, \tilde{f}_{n}, G_{1, n}, G_{2, n}$ and $G_{3, n}$ are defined in (4.9). We will prove (4.10), which gives a contradiction with (4.5). Using (2.28), we find

$$
\operatorname{Re}\left\langle\lambda_{n}^{j}\left(i \lambda_{n} I-\mathcal{A}\right) U_{n}, U_{n}\right\rangle_{\mathcal{H}}=\operatorname{Re}\left(i \lambda_{n}^{j+1}\left\|U_{n}\right\|_{\mathcal{H}}^{2}-\lambda_{n}^{j}\left\langle\mathcal{A} U_{n}, U_{n}\right\rangle_{\mathcal{H}}\right)=\frac{-\lambda_{n}^{j}}{2} \int_{0}^{\infty} g^{\prime}(s)\left\|\eta_{n x}\right\|^{2} \mathrm{~d} s
$$

So (4.5) and (5.3) imply that

$$
\lambda_{n}^{j} \int_{0}^{\infty} g^{\prime}(s)\left\|\eta_{n x}\right\|^{2} \mathrm{~d} s \longrightarrow 0
$$

Then multiplying (4.12) by $\left|\lambda_{n}\right|^{j}$ and using (5.5), we obtain

$$
\lambda_{n}^{j} \int_{0}^{\infty} g(s)\left\|\eta_{n x}\right\|^{2} \mathrm{~d} s \longrightarrow 0
$$

hence

$$
\lambda_{n}^{\frac{j}{2}} \eta_{n} \longrightarrow 0 \text { in } L_{g}
$$


Using (4.5) and (4.6), we get from the last limit in (5.4) that

$$
\lambda_{n}^{\frac{j}{2}-1}\left\langle\left(i \lambda_{n} \eta_{n}+\eta_{n s}-i \lambda_{n} f_{n}+i \lambda_{n} f_{n}-\tilde{f}_{n}\right), i f_{n}\right\rangle_{L_{g}} \longrightarrow 0
$$

On the other hand, using (5.4) 1 in case (2.2), (5.4) 3 in case (2.3) and (5.4) $)_{5}$ in case (2.4), we get

$$
\lambda_{n}^{\frac{j}{2}-1}\left\langle\left(i \lambda_{n} f_{n}-\tilde{f}_{n}\right), i f_{n}\right\rangle_{L_{g}} \longrightarrow 0
$$

So (5.7) and (5.8) lead to

$$
\left\langle\lambda_{n}^{\frac{j}{2}} \eta_{n}, f_{n}\right\rangle_{L_{g}}-i \lambda_{n}^{\frac{j}{2}-1}\left\langle\eta_{n s}, f_{n}\right\rangle_{L_{g}}-\lambda_{n}^{\frac{j}{2}}\left\langle f_{n}, f_{n}\right\rangle_{L_{g}} \longrightarrow 0
$$

We note that

$$
-\lambda_{n}^{\frac{j}{2}}\left\langle f_{n}, f_{n}\right\rangle_{L_{g}}=-g_{0} \lambda_{n}^{\frac{j}{2}}\left\|f_{n x}\right\|^{2}
$$

On the other hand, (4.5) and (5.6) imply that

$$
\left\langle\lambda_{n}^{\frac{j}{2}} \eta_{n}, f_{n}\right\rangle_{L_{g}} \longrightarrow 0
$$

Moreover, using (4.20), we find

$$
\left|\lambda_{n}^{\frac{j}{2}-1}\left\langle\eta_{n s}, f_{n}\right\rangle_{L_{g}}\right| \leq \sqrt{\beta_{1} g(0)}\left|\lambda_{n}\right|^{\frac{j}{2}-1}\left\|f_{n x}\right\|\left\|\eta_{n}\right\|_{L_{g}},
$$

and then, according to (4.5), (4.6) and (5.6),

$$
\lambda_{n}^{\frac{j}{2}-1}\left\langle\eta_{n s}, f_{n}\right\rangle_{L_{g}} \longrightarrow 0
$$

Consequently, (5.9)-(5.12) and since $g_{0}>0$ (hypothesis (H)) lead to

$$
\left|\lambda_{n}\right|^{\frac{j}{4}} f_{n x} \longrightarrow 0 \text { in } L^{2}(0,1) .
$$

Moreover, because $f_{n}$ vanishes at $x=1$ (in case (2.2)) or at $x=0$ (in cases (2.3) and (2.4)), then

$$
\left|\lambda_{n}\right|^{\frac{j}{4}} f_{n} \longrightarrow 0 \text { in } L^{2}(0,1)
$$

Multiplying (5.4) $)_{1}$ in case (2.2), (5.4) 3 in case (2.3) and (5.4) 5 in case (2.4) by $\left|\lambda_{n}\right|^{-\frac{3 j}{4}-1}$ and using (4.6), we conclude from (5.13) and (5.14) that

$$
\left|\lambda_{n}\right|^{\frac{j}{4}-1} \tilde{f}_{n x} \rightarrow 0 \text { in } L^{2}(0,1)
$$


and

$$
\left|\lambda_{n}\right|^{\frac{j}{4}-1} \tilde{f}_{n} \longrightarrow 0 \text { in } L^{2}(0,1)
$$

Multiplying (5.4) $)_{1},(5.4)_{3}$ and (5.4) $)_{5}$ by $\lambda_{n}^{-j-1}$, and using (4.5) and (4.6), we obtain (4.25). Multiplying $(5.4)_{1},(5.4)_{3}$ and $(5.4)_{5}$ by $\lambda_{n}^{-j}$, multiplying $(5.4)_{2},(5.4)_{4}$ and (5.4) 6 by $\lambda_{n}^{-j-1}$ and using (4.5) and (4.6), we get, respectively,

$$
\left(\lambda_{n} \varphi_{n}\right)_{n},\left(\lambda_{n} \psi_{n}\right)_{n} \text { and }\left(\lambda_{n} w_{n}\right)_{n} \text { are bounded in } L^{2}(0,1)
$$

and

$$
\left(\frac{1}{\lambda_{n}}\left(k \varphi_{n}-G_{1, n}\right)_{x x}\right)_{n},\left(\frac{1}{\lambda_{n}}\left(b \psi_{n}-G_{2, n}\right)_{x x}\right)_{n} \text { and }\left(\frac{1}{\lambda_{n}}\left(k_{0} w_{n}-G_{3, n}\right)_{x x}\right)_{n} \text { are bounded in } L^{2}(0,1) \text {. }
$$

Now, we distinguish the cases (2.2), (2.3) and (2.4). We will apply several multipliers and use some arguments of Guesmia (2017b).

5.1. Case (2.2) $\left(j=4, f_{n}=\varphi_{n}, \tilde{f}_{n}=\tilde{\varphi}_{n}\right.$ and $\left.G_{2, n}=G_{3, n}=0\right)$

Taking the inner product of (5.4) 2 with $\frac{i \tilde{\varphi}_{n}}{\lambda_{n}^{3}}$ in $L^{2}(0,1)$, using (4.5), (4.6) and (4.9), integrating by parts and using the boundary conditions, we have

$$
\rho_{1}\left\|\lambda_{n} \tilde{\varphi}_{n}\right\|^{2}+\left\langle k \lambda_{n}\left(\varphi_{n x}+\psi_{n}+w_{n}\right), i \tilde{\varphi}_{n x}\right\rangle-\left\langle\lambda_{n}\left(g_{0} f_{n x}-\int_{0}^{\infty} g \eta_{n x} \mathrm{~d} s\right), i \tilde{\varphi}_{n x}\right\rangle \rightarrow 0 .
$$

So, using (4.5), (5.6), (5.13), (5.15), (5.17) and (5.19), we deduce that

$$
\lambda_{n} \tilde{\varphi}_{n} \longrightarrow 0 \text { in } L^{2}(0,1),
$$

and by (4.6) and (5.4) 1 , we find

$$
\lambda_{n}^{2} \varphi_{n} \longrightarrow 0 \text { in } L^{2}(0,1)
$$

Taking the inner product of $(5.4)_{2}$ with $\frac{1}{\lambda_{n}^{4}}\left(k \psi_{n x}+k w_{n x}\right)$ in $L^{2}(0,1)$ and using (4.5), (4.6) and (4.9), we arrive at

$$
\begin{aligned}
\rho_{1}\left\langle i \lambda_{n} \tilde{\varphi}_{n},\left(k \psi_{n x}+k w_{n x}\right)\right\rangle+ & \left(g_{0}-k\right)\left\langle\varphi_{n x x},\left(k \psi_{n x}+k w_{n x}\right)\right\rangle \\
& -\left\|k \psi_{n x}+k w_{n x}\right\|^{2}-\left\langle\int_{0}^{\infty} g(s) \eta_{n x x} \mathrm{~d} s,\left(k \psi_{n x}+k w_{n x}\right)\right\rangle \rightarrow 0
\end{aligned}
$$

Integrating by parts and using the boundary conditions, we find

$$
\left\langle\varphi_{n x x},\left(k \psi_{n x}+k w_{n x}\right)\right\rangle=-\left\langle\lambda_{n} \varphi_{n x},\left(k \frac{\psi_{n x x}}{\lambda_{n}}+k \frac{w_{n x x}}{\lambda_{n}}\right)\right\rangle
$$


and

$$
\left\langle\int_{0}^{\infty} g(s) \eta_{n x x} \mathrm{~d} s,\left(k \psi_{n x}+k w_{n x}\right)\right\rangle=-\left\langle\lambda_{n} \int_{0}^{\infty} g(s) \eta_{n x} \mathrm{~d} s,\left(k \frac{\psi_{n x x}}{\lambda_{n}}+k \frac{w_{n x x}}{\lambda_{n}}\right)\right\rangle .
$$

Multiplying (5.4) $)_{4}$ and (5.4) 6 by $\frac{1}{\lambda_{n}^{5}}$ and using (4.6) and (4.9), we obtain

$$
\left\{\begin{array}{l}
i \rho_{2} \tilde{\psi}_{n}-b \frac{\psi_{n x x}}{\lambda_{n}}+\frac{k}{\lambda_{n}}\left(\varphi_{n x}+\psi_{n}+w_{n}\right) \rightarrow 0 \text { in } L^{2}(0,1) \\
i \rho_{3} \tilde{w}_{n}-k_{0} \frac{w_{n x x}}{\lambda_{n}}+\frac{k}{\lambda_{n}}\left(\varphi_{n x}+\psi_{n}+w_{n}\right)+\frac{\delta}{\lambda_{n}} w_{n} \rightarrow 0 \text { in } L^{2}(0,1)
\end{array}\right.
$$

Exploiting (4.5) and (4.6), we get

$$
\left(\frac{1}{\lambda_{n}} \psi_{n x x}\right)_{n} \text { and }\left(\frac{1}{\lambda_{n}} w_{n x x}\right)_{n} \text { are bounded in } L^{2}(0,1) .
$$

Then, using (5.6), (5.13), (5.23), (5.24) and (5.25), we deduce that

$$
\left\langle\varphi_{n x x},\left(k \psi_{n x}+k w_{n x}\right)\right\rangle \rightarrow 0 \text { and }\left\langle\int_{0}^{\infty} g(s) \eta_{n x x} \mathrm{~d} s,\left(k \psi_{n x}+k w_{n x}\right)\right\rangle \rightarrow 0,
$$

so, exploiting (4.5), (5.20), (5.22) and (5.26), we have

$$
\psi_{n x}+w_{n x} \rightarrow 0 \text { in } L^{2}(0,1)
$$

Taking the inner product of $(5.4)_{4}$ with $\frac{\psi_{n}}{\lambda_{n}^{4}}$ in $L^{2}(0,1)$, using (4.5), (4.6) and (4.9), integrating by parts and using the boundary conditions, we obtain

$$
-\rho_{2}\left\langle\tilde{\psi}_{n},\left(i \lambda_{n} \psi_{n}-\tilde{\psi}_{n}\right)\right\rangle-\rho_{2}\left\|\tilde{\psi}_{n}\right\|^{2}+b\left\|\psi_{n x}\right\|^{2}+k\left\langle\left(\varphi_{n x}+\psi_{n}+w_{n}\right), \psi_{n}\right\rangle \rightarrow 0
$$

then, using (4.5), (4.6), (4.25) and (5.4) 3 , we find

$$
b\left\|\psi_{n x}\right\|^{2}-\rho_{2}\left\|\tilde{\psi}_{n}\right\|^{2} \rightarrow 0
$$

Taking the inner product of $(5.4)_{6}$ with $\frac{w_{n}}{\lambda_{n}^{4}}$ in $L^{2}(0,1)$, using (4.5), (4.6) and (4.9), integrating by parts and using the boundary conditions, we observe that

$$
-\rho_{3}\left\langle\tilde{w}_{n},\left(i \lambda_{n} w_{n}-\tilde{w}_{n}\right)\right\rangle-\rho_{3}\left\|\tilde{w}_{n}\right\|^{2}+k_{0}\left\|w_{n x}\right\|^{2}+k\left\langle\left(\varphi_{n x}+\psi_{n}+w_{n}\right), w_{n}\right\rangle+\delta\left\|w_{n}\right\|^{2} \rightarrow 0 .
$$

By (4.5), (4.6), (4.25) and (5.4) $)_{5}$ we deduce that

$$
k_{0}\left\|w_{n x}\right\|^{2}-\rho_{3}\left\|\tilde{w}_{n}\right\|^{2} \rightarrow 0 .
$$


Taking the inner product of (5.4) $)_{4}$ with $\frac{w_{n}}{\lambda_{n}^{4}}$ and (5.4) 6 with $\frac{\psi_{n}}{\lambda_{n}^{4}}$ in $L^{2}(0,1)$, using (4.5), (4.6) and (4.9), integrating by parts and using the boundary conditions, we obtain

$$
-\rho_{2}\left\langle\tilde{\psi}_{n},\left(i \lambda_{n} w_{n}-\tilde{w}_{n}\right)\right\rangle-\rho_{2}\left\langle\tilde{\psi}_{n}, \tilde{w}_{n}\right\rangle+b\left\langle\psi_{n x}, w_{n x}\right\rangle+k\left\langle\left(\varphi_{n x}+\psi_{n}+w_{n}\right), w_{n}\right\rangle \rightarrow 0
$$

and

$$
-\rho_{3}\left\langle\tilde{w}_{n},\left(i \lambda_{n} \psi_{n}-\tilde{\psi}_{n}\right)\right\rangle-\rho_{3}\left\langle\tilde{w}_{n}, \tilde{\psi}_{n}\right\rangle+k_{0}\left\langle w_{n x}, \psi_{n x}\right\rangle+\left\langle k\left(\varphi_{n x}+\psi_{n}+w_{n}\right)+\delta w_{n}, \psi_{n}\right\rangle \rightarrow 0,
$$

then, using (4.5), (4.6), (4.25), (5.4) 3 and (5.4) 5 , we obtain

$$
-\rho_{2}\left\langle\tilde{\psi}_{n}, \tilde{w}_{n}\right\rangle+b\left\langle\psi_{n x}, w_{n x}\right\rangle \rightarrow 0 \quad \text { and } \quad-\rho_{3}\left\langle\tilde{\psi}_{n}, \tilde{w}_{n}\right\rangle+k_{0}\left\langle\psi_{n x}, w_{n x}\right\rangle \rightarrow 0
$$

which imply that

$$
\left(\frac{\rho_{2}}{b}-\frac{\rho_{3}}{k_{0}}\right)\left\langle\tilde{\psi}_{n}, \tilde{w}_{n}\right\rangle \rightarrow 0 \text { and }\left(\frac{b}{\rho_{2}}-\frac{k_{0}}{\rho_{3}}\right)\left\langle\psi_{n x}, w_{n x}\right\rangle \rightarrow 0 .
$$

We distinguish two cases.

Case 1: $\frac{b}{\rho_{2}} \neq \frac{k_{0}}{\rho_{3}}$. From (5.30), we see that

$$
\left\langle\tilde{\psi}_{n}, \tilde{w}_{n}\right\rangle \rightarrow 0 \text { and }\left\langle\psi_{n x}, w_{n x}\right\rangle \rightarrow 0
$$

Therefore, taking the inner product in $L^{2}(0,1)$ of $(5.27)$, first, with $\psi_{n x}$, and, second, with $w_{n x}$, we obtain

$$
\psi_{n x} \rightarrow 0 \text { and } \quad w_{n x} \rightarrow 0 \text { in } L^{2}(0,1)
$$

and then, by (5.28), (5.29) and (5.32), we find

$$
\tilde{\psi}_{n} \rightarrow 0 \text { and } \quad \tilde{w}_{n} \rightarrow 0 \text { in } L^{2}(0,1)
$$

Finally, combining (4.25), (5.6), (5.13), (5.16), (5.32) and (5.33), we get (4.10), which is a contradiction with (4.5), so (5.2) holds. Consequently, (5.1) in case (2.2) with $j=4$ is satisfied.

Case 2: $\frac{b}{\rho_{2}}=\frac{k_{0}}{\rho_{3}}$. Using (4.6) and (4.9), and multiplying (5.4) $4_{4}$ and (5.4) 6 by $\frac{1}{b \lambda_{n}^{2}}$ and $\frac{1}{k_{0} \lambda_{n}^{2}}$, respectively, we obtain

$$
\left\{\begin{array}{l}
\lambda_{n}^{2}\left[-\frac{i \rho_{2}}{b} \lambda_{n}\left(i \lambda_{n} \psi_{n}-\tilde{\psi}_{n}\right)-\frac{\rho_{2}}{b} \lambda_{n}^{2} \psi_{n}-\psi_{n x x}+\frac{k}{b}\left(\varphi_{n x}+\psi_{n}+w_{n}\right)\right] \rightarrow 0 \text { in } L^{2}(0,1), \\
\lambda_{n}^{2}\left[-\frac{i \rho_{2}}{b} \lambda_{n}\left(i \lambda_{n} w_{n}-\tilde{w}_{n}\right)-\frac{\rho_{2}}{b} \lambda_{n}^{2} w_{n}-w_{n x x}+\frac{k}{k_{0}}\left(\varphi_{n x}+\psi_{n}+w_{n}\right)+\frac{\delta}{k_{0}} w_{n}\right] \rightarrow 0 \text { in } L^{2}(0,1),
\end{array}\right.
$$


so, using $(5.4)_{3}$ and $(5.4)_{5}$, we find

$$
\left\{\begin{array}{l}
\lambda_{n}^{2}\left[-\frac{\rho_{2}}{b} \lambda_{n}^{2} \psi_{n}-\psi_{n x x}+\frac{k}{b}\left(\varphi_{n x}+\psi_{n}+w_{n}\right)\right] \rightarrow 0 \text { in } L^{2}(0,1) \\
\lambda_{n}^{2}\left[-\frac{\rho_{2}}{b} \lambda_{n}^{2} w_{n}-w_{n x x}+\frac{k}{k_{0}}\left(\varphi_{n x}+\psi_{n}+w_{n}\right)+\frac{\delta}{k_{0}} w_{n}\right] \rightarrow 0 \text { in } L^{2}(0,1)
\end{array}\right.
$$

Then, multiplying $(5.34)_{1}$ and $(5.34)_{2}$ by $\frac{1}{\lambda_{n}^{2}}$, using (4.6), (4.25) and (5.13), we get

$$
\frac{\rho_{2}}{b} \lambda_{n}^{2} \psi_{n}+\psi_{n x x} \rightarrow 0 \text { in } L^{2}(0,1) \quad \text { and } \quad \frac{\rho_{2}}{b} \lambda_{n}^{2} w_{n}+w_{n x x} \rightarrow 0 \text { in } L^{2}(0,1)
$$

The sum and difference of $(5.35)_{1}$ and $(5.35)_{2}$ give

$$
\left\{\begin{array}{l}
\frac{\rho_{2}}{b} \lambda_{n}^{2}\left(\psi_{n}+w_{n}\right)+\psi_{n x x}+w_{n x x} \rightarrow 0 \text { in } L^{2}(0,1) \\
\frac{\rho_{2}}{b} \lambda_{n}^{2}\left(\psi_{n}-w_{n}\right)+\psi_{n x x}-w_{n x x} \rightarrow 0 \text { in } L^{2}(0,1)
\end{array}\right.
$$

Taking the inner product in $L^{2}(0,1)$ of $(5.36)_{1}$ and $(5.36)_{2}$ with $\psi_{n}+w_{n}$, using (4.5), integrating by parts and using the boundary conditions, we get

$$
\frac{\rho_{2}}{b}\left\|\lambda_{n} \psi_{n}+\lambda_{n} w_{n}\right\|^{2}-\left\|\psi_{n x}+w_{n x}\right\|_{L^{2}(0,1)}^{2} \rightarrow 0
$$

and

$$
\frac{\rho_{2}}{b}\left\langle\lambda_{n}^{2}\left(\psi_{n}-w_{n}\right),\left(\psi_{n}+w_{n}\right)\right\rangle-\left\langle\left(\psi_{n x}-w_{n x}\right),\left(\psi_{n x}+w_{n x}\right)\right\rangle \rightarrow 0
$$

then, using (4.5) and (5.27), we obtain

$$
\lambda_{n} \psi_{n}+\lambda_{n} w_{n} \rightarrow 0 \text { in } L^{2}(0,1) \quad \text { and } \quad\left\|\lambda_{n} \psi_{n}\right\|^{2}-\left\|\lambda_{n} w_{n}\right\|^{2} \rightarrow 0
$$

Taking the inner product in $L^{2}(0,1)$ of $(5.34)_{1}$ and $(5.34)_{2}$ with $w_{n}$ and $\psi_{n}$, respectively, integrating by parts and using (4.5) and the boundary conditions, we get

$$
-\frac{\rho_{2}}{b} \lambda_{n}^{4}\left\langle\psi_{n}, w_{n}\right\rangle+\lambda_{n}^{2}\left\langle\psi_{n x}, w_{n x}\right\rangle-\frac{k}{b}\left\langle\lambda_{n}^{2} \varphi_{n}, w_{n x}\right\rangle+\frac{k}{b}\left\langle\lambda_{n} \psi_{n}, \lambda_{n} w_{n}\right\rangle+\frac{k}{b}\left\|\lambda_{n} w_{n}\right\|^{2} \rightarrow 0
$$

and

$$
-\frac{\rho_{2}}{b} \lambda_{n}^{4}\left\langle\psi_{n}, w_{n}\right\rangle+\lambda_{n}^{2}\left\langle\psi_{n x}, w_{n x}\right\rangle-\frac{k}{k_{0}}\left\langle\psi_{n x}, \lambda_{n}^{2} \varphi_{n}\right\rangle+\frac{k}{k_{0}}\left\|\lambda_{n} \psi_{n}\right\|^{2}+\frac{k+\delta}{k_{0}}\left\langle\lambda_{n} \psi_{n}, \lambda_{n} w_{n}\right\rangle \rightarrow 0
$$

Then, taking the difference between (5.38) and (5.39) and using (4.5) and (5.21), we find

$$
\frac{k}{b}\left\|\lambda_{n} w_{n}\right\|^{2}-\frac{k}{k_{0}}\left\|\lambda_{n} \psi_{n}\right\|^{2}+\left(\frac{k}{b}-\frac{k+\delta}{k_{0}}\right)\left\langle\lambda_{n} \psi_{n}, \lambda_{n} w_{n}\right\rangle \rightarrow 0
$$


By taking the inner product in $L^{2}(0,1)$ of $(5.37)_{1}$ with $\lambda_{n} \psi_{n}$, and using (5.17), we have

$$
\left\|\lambda_{n} \psi_{n}\right\|^{2}+\left\langle\lambda_{n} \psi_{n}, \lambda_{n} w_{n}\right\rangle \rightarrow 0
$$

Multiplying (5.37) 2 by $\frac{k}{b}$ and adding (5.40), we get

$$
k\left(\frac{1}{b}-\frac{1}{k_{0}}\right)\left\|\lambda_{n} \psi_{n}\right\|^{2}+\left(\frac{k}{b}-\frac{k+\delta}{k_{0}}\right)\left\langle\lambda_{n} \psi_{n}, \lambda_{n} w_{n}\right\rangle_{L^{2}(0,1)} \rightarrow 0
$$

Therefore, multiplying (5.41) by $\frac{k+\delta}{k_{0}}-\frac{k}{b}$ and adding (5.42), we obtain

$$
\frac{\delta}{k_{0}}\left\|\lambda_{n} \psi_{n}\right\|^{2} \rightarrow 0
$$

Then

$$
\lambda_{n} \psi_{n} \rightarrow 0 \text { in } L^{2}(0,1)
$$

and, using (5.37) 1 ,

$$
\lambda_{n} w_{n} \rightarrow 0 \text { in } L^{2}(0,1) .
$$

Using (4.6), (5.4) $3,(5.4)_{5},(5.43)$ and (5.44), we deduce that

$$
\tilde{\psi}_{n} \rightarrow 0 \text { in } L^{2}(0,1) \text { and } \quad \tilde{w}_{n} \rightarrow 0 \text { in } L^{2}(0,1) .
$$

Taking the inner product in $L^{2}(0,1)$ of $(5.35)_{1}$ and $(5.35)_{2}$ with $\psi_{n}$ and $w_{n}$, respectively, integrating by parts and using (4.5) and the boundary conditions, we get

$$
\frac{\rho_{2}}{b}\left\|\lambda_{n} \psi_{n}\right\|^{2}-\left\|\psi_{n x}\right\|^{2} \rightarrow 0 \quad \text { and } \quad \frac{\rho_{2}}{b}\left\|\lambda_{n} w_{n}\right\|^{2}-\left\|w_{n x}\right\|^{2} \rightarrow 0,
$$

then, from (5.43) and (5.44), we conclude that

$$
\psi_{n x} \rightarrow 0 \text { in } L^{2}(0,1) \quad \text { and } \quad w_{n x} \rightarrow 0 \text { in } L^{2}(0,1)
$$

Finally, (4.25), (5.6), (5.13), (5.16), (5.45) and (5.46) imply (4.10), which is a contradiction with (4.5). Thus, in both cases $\frac{b}{\rho_{2}} \neq \frac{k_{0}}{\rho_{3}}$ and $\frac{b}{\rho_{2}}=\frac{k_{0}}{\rho_{3}}$, (5.2) holds, and so (5.1) is satisfied in case (2.2) with $j=4$.

5.2. $\operatorname{Case}(2.3)\left(j=10, f_{n}=\psi_{n}, \tilde{f}_{n}=\tilde{\psi}_{n}\right.$ and $\left.G_{1, n}=G_{3, n}=0\right)$

Taking the inner product of $(5.4)_{4}$ with $\frac{1}{\lambda_{n}^{10}} \varphi_{n x}$ in $L^{2}(0,1)$, integrating by parts and using (4.5), (4.6) and the boundary conditions, we get

$$
i \rho_{2}\left\langle\lambda_{n} \tilde{\psi}_{n}, \varphi_{n x}\right\rangle+\left\langle k\left(\psi_{n}+w_{n}\right), \varphi_{n x}\right\rangle+k\left\|\varphi_{n x}\right\|^{2}+\left\langle\lambda_{n}\left(b \psi_{n x}-G_{2, n x}\right), \frac{1}{\lambda_{n}} \varphi_{n x x}\right\rangle \rightarrow 0,
$$


then, using (4.25), (5.6), (5.13), (5.16) and (5.18), we deduce that

$$
\varphi_{n x} \rightarrow 0 \text { in } L^{2}(0,1)
$$

which implies that, using (4.6) and (5.4) 1 ,

$$
\frac{1}{\lambda_{n}} \tilde{\varphi}_{n x} \rightarrow 0 \text { in } L^{2}(0,1) .
$$

Dividing (5.4) $)_{4}$ by $\lambda_{n}^{10}$ and using (4.25), (5.16) and (5.47), we deduce that

$$
b \psi_{n x x}-G_{2, n x x} \rightarrow 0 \text { in } L^{2}(0,1) .
$$

Taking the inner product of $(5.4)_{4}$ with $\frac{\varphi_{n x}}{\lambda_{n}^{9}}$ in $L^{2}(0,1)$, integrating by parts and using (4.5), (4.6) and the boundary conditions, we get

$$
\begin{aligned}
-\rho_{2}\left\langle\tilde{\psi}_{n}, \lambda_{n}\left(i \lambda_{n} \varphi_{n x}-\right.\right. & \left.\left.\tilde{\varphi}_{n x}\right)\right\rangle+\rho_{2}\left\langle\lambda_{n} \tilde{\psi}_{n x}, \tilde{\varphi}_{n}\right\rangle \\
& +\left\langle\lambda_{n}^{2}\left(b \psi_{n x}-G_{2, n x}\right), \frac{\varphi_{n x x}}{\lambda_{n}}\right\rangle+k \lambda_{n}\left\|\varphi_{n x}\right\|^{2}+k\left\langle\lambda_{n}\left(\psi_{n}+w_{n}\right), \varphi_{n x}\right\rangle \longrightarrow 0 ;
\end{aligned}
$$

hence, using (5.4) 1 , (5.6), (5.13), (5.15), (5.17), (5.18) and (5.47), we obtain

$$
\left|\lambda_{n}\right|^{\frac{1}{2}} \varphi_{n x} \longrightarrow 0 \text { in } L^{2}(0,1)
$$

Therefore, according to the boundary conditions, (5.50) leads to

$$
\left|\lambda_{n}\right|^{\frac{1}{2}} \varphi_{n} \longrightarrow 0 \text { in } L^{2}(0,1)
$$

Taking the inner product of $(5.4)_{2}$ with $\frac{\varphi_{n}}{\lambda_{n}^{9}}$ in $L^{2}(0,1)$, integrating by parts and using (4.5), (4.6) and the boundary conditions, we get

$$
-\rho_{1}\left\langle\tilde{\varphi}_{n}, \lambda_{n}\left(i \lambda_{n} \varphi_{n}-\tilde{\varphi}_{n}\right)\right\rangle-\rho_{1} \lambda_{n}\left\|\tilde{\varphi}_{n}\right\|^{2}+k \lambda_{n}\left\|\varphi_{n x}\right\|^{2}+k\left\langle\left(\lambda_{n} \psi_{n}+\lambda_{n} w_{n}\right), \varphi_{n x}\right\rangle \longrightarrow 0,
$$

so, using $(5.4)_{1},(5.17),(5.47)$ and $(5.50)$, we deduce that

$$
\left|\lambda_{n}\right|^{\frac{1}{2}} \tilde{\varphi}_{n} \longrightarrow 0 \text { in } L^{2}(0,1)
$$

which implies that, using (4.6) and (5.4) 1 ,

$$
\left|\lambda_{n}\right|^{\frac{3}{2}} \varphi_{n} \longrightarrow 0 \text { in } L^{2}(0,1) .
$$


Multiplying (5.4) 2 by $\left|\lambda_{n}\right|^{-10-\frac{1}{2}}$ and using (4.6), we get

$$
i \rho_{1} \frac{\lambda_{n}}{\left|\lambda_{n}\right|^{\frac{1}{2}}} \tilde{\varphi}_{n}-k \frac{\varphi_{n x x}}{\left|\lambda_{n}\right|^{\frac{1}{2}}}-k \frac{\psi_{n x}}{\left|\lambda_{n}\right|^{\frac{1}{2}}}-k \frac{w_{n x}}{\left|\lambda_{n}\right|^{\frac{1}{2}}} \longrightarrow 0 \text { in } L^{2}(0,1),
$$

then, using (4.5), (4.6) and (5.52), we obtain

$$
\frac{\varphi_{n x x}}{\left|\lambda_{n}\right|^{\frac{1}{2}}} \longrightarrow 0 \text { in } L^{2}(0,1)
$$

Integrating by parts and using the boundary conditions, we have

$$
\begin{aligned}
\lambda_{n}\left\langle\left(\varphi_{n x}+\psi_{n}+w_{n}\right)_{x}, \tilde{\varphi}_{n}\right\rangle= & -\lambda_{n}\left\langle\left(\varphi_{n x}+\psi_{n}+w_{n}\right), \tilde{\varphi}_{n x}\right\rangle \\
= & -\frac{1}{k}\left\langle\lambda_{n}^{2}\left[i \lambda_{n} \rho_{2} \tilde{\psi}_{n}-b \psi_{n x x}+k\left(\varphi_{n x}+\psi_{n}+w_{n}\right)+G_{2, n x x}\right], \frac{\tilde{\varphi}_{n x}}{\lambda_{n}}\right\rangle \\
& -\frac{1}{k}\left\langle i \lambda_{n} \rho_{2} \tilde{\psi}_{n}, \lambda_{n}\left(i \lambda_{n} \varphi_{n x}-\tilde{\varphi}_{n x}\right)\right\rangle+\frac{1}{k}\left\langle\left|\lambda_{n}\right|^{\frac{5}{2}}\left(b \psi_{n x}-G_{2, n x}\right), \frac{i \varphi_{n x x}}{\left.\left|\lambda_{n}\right|^{\frac{1}{2}}\right\rangle}\right. \\
& +\frac{1}{k}\left\langle\left(b \psi_{n x x}-G_{2, n x x}\right), \lambda_{n}\left(i \lambda_{n} \varphi_{n x}-\tilde{\varphi}_{n x}\right)\right\rangle-\frac{\lambda_{n}^{3}}{k\left|\lambda_{n}\right|^{3}}\left\langle i \rho_{2}\left|\lambda_{n}\right|^{\frac{3}{2}} \tilde{\psi}_{n x}, i\left|\lambda_{n}\right|^{\frac{3}{2}} \varphi_{n}\right\rangle,
\end{aligned}
$$

then, using $(5.4)_{1},(5.4)_{4},(5.6),(5.13),(5.15),(5.16),(5.48),(5.49),(5.53)$ and (5.54), we find

$$
\lambda_{n}\left\langle\left(\varphi_{n x}+\psi_{n}+w_{n}\right)_{x}, \tilde{\varphi}_{n}\right\rangle \longrightarrow 0
$$

Taking the inner product of $(5.4)_{2}$ with $\frac{\tilde{\varphi}_{n}}{\lambda_{n}^{9}}$ in $L^{2}(0,1)$ and using (4.5) and (4.6), we get

$$
\rho_{1} i\left\|\lambda_{n} \tilde{\varphi}_{n}\right\|^{2}-k \lambda_{n}\left\langle\left(\varphi_{n x}+\psi_{n}+w_{n}\right)_{x}, \tilde{\varphi}_{n}\right\rangle \longrightarrow 0
$$

then, using (5.55), we obtain

$$
\lambda_{n} \tilde{\varphi}_{n} \longrightarrow 0 \text { in } L^{2}(0,1)
$$

and so (5.4) $)_{1}$ implies that

$$
\lambda_{n}^{2} \varphi_{n} \longrightarrow 0 \text { in } L^{2}(0,1) .
$$

Taking the inner product of $(5.4)_{2}$ with $\frac{\varphi_{n}}{\lambda_{n}^{8}}$ in $L^{2}(0,1)$, integrating by parts and using (4.5) and (4.6), we get

$$
k\left\|\lambda_{n} \varphi_{n x}\right\|^{2}+\left\langle i \rho_{1} \lambda_{n} \tilde{\varphi}_{n}-k\left(\psi_{n x}+w_{n x}\right), \lambda_{n}^{2} \varphi_{n}\right\rangle \longrightarrow 0
$$


therefore, according to (5.56) and (5.57), we find

$$
\lambda_{n} \varphi_{n x} \longrightarrow 0 \text { in } L^{2}(0,1)
$$

Taking the inner product of $(5.4)_{2}$ with $\frac{w_{n x}}{\lambda_{n}^{10}}$ in $L^{2}(0,1)$ and using (4.5) and (4.6), we get

$$
\rho_{1}\left\langle i \lambda_{n} \tilde{\varphi}_{n}, w_{n x}\right\rangle-k\left\langle\varphi_{n x x}, w_{n x}\right\rangle-k\left\|w_{n x}\right\|^{2}-k\left\langle\psi_{n x}, w_{n x}\right\rangle \rightarrow 0
$$

then, integrating by parts and using the boundary conditions, we obtain

$$
\rho_{1}\left\langle i \lambda_{n} \tilde{\varphi}_{n}, w_{n x}\right\rangle+k\left\langle\lambda_{n} \varphi_{n x}, \frac{w_{n x x}}{\lambda_{n}}\right\rangle-k\left\|w_{n x}\right\|^{2}-k\left\langle\psi_{n x}, w_{n x}\right\rangle \rightarrow 0
$$

so, using (5.13), (5.18), (5.56) and (5.58), we deduce that

$$
w_{n x} \longrightarrow 0 \text { in } L^{2}(0,1)
$$

Taking the inner product of $(5.4)_{6}$ with $\frac{w_{n}}{\lambda_{n}^{10}}$ in $L^{2}(0,1)$, integrating by parts and using (4.5) and (4.6) and the boundary conditions, we get

$$
-\rho_{3}\left\langle\tilde{w}_{n},\left(i \lambda_{n} w_{n}-\tilde{w}_{n}\right)\right\rangle-\rho_{3}\left\|\tilde{w}_{n}\right\|^{2}+k_{0}\left\|w_{n x}\right\|^{2}+\left\langle k\left(\varphi_{n x}+\psi_{n}+w_{n}\right)+\delta w_{n}, w_{n}\right\rangle \longrightarrow 0 ;
$$

hence, using $(5.4)_{5},(4.25)$ and (5.59), we get

$$
\tilde{w}_{n} \longrightarrow 0 \text { in } L^{2}(0,1)
$$

A combination of the limits (4.25), (5.6), (5.13), (5.16), (5.47), (5.52), (5.59) and (5.60) leads to (4.10), which is a contradiction with (4.5). Consequently, (5.2) holds, and so (5.1) is satisfied in case (2.3) with $j=10$.

5.3. Case (2.4) $\left(j=10, f_{n}=w_{n}, \tilde{f}_{n}=\tilde{w}_{n}\right.$ and $\left.G_{1, n}=G_{2, n}=0\right)$

The proof is very similar to the one given in Section 5.2. Taking the inner product of $(5.4)_{6}$ with $\frac{1}{\lambda_{n}^{10}} \varphi_{n x}$ in $L^{2}(0,1)$, integrating by parts and using (4.5), (4.6) and the boundary conditions, we get

$$
i \rho_{3}\left\langle\lambda_{n} \tilde{w}_{n}, \varphi_{n x}\right\rangle+\left\langle k\left(\psi_{n}+w_{n}\right), \varphi_{n x}\right\rangle+k\left\|\varphi_{n x}\right\|^{2}+\left\langle\lambda_{n}\left(k_{0} w_{n x}-G_{3, n x}\right), \frac{1}{\lambda_{n}} \varphi_{n x x}\right\rangle \rightarrow 0,
$$

then, using (4.25), (5.6), (5.13), (5.16) and (5.18), we deduce (5.47), and so (5.48) holds.

Dividing $(5.4)_{6}$ by $\lambda_{n}^{10}$ and using (4.6), (4.25), (5.16) and (5.47), we deduce that

$$
k_{0} w_{n x x}-G_{3, n x x} \rightarrow 0 \text { in } L^{2}(0,1) .
$$


Taking the inner product of $(5.4)_{6}$ with $\frac{\varphi_{n x}}{\lambda_{n}^{9}}$ in $L^{2}(0,1)$, integrating by parts and using (4.5), (4.6) and the boundary conditions, we get

$$
\begin{aligned}
& -\rho_{3}\left\langle\tilde{w}_{n}, \lambda_{n}\left(i \lambda_{n} \varphi_{n x}-\tilde{\varphi}_{n x}\right)\right\rangle+\rho_{3}\left\langle\lambda_{n} \tilde{w}_{n x}, \tilde{\varphi}_{n}\right\rangle \\
& \quad+\left\langle\lambda_{n}^{2}\left(k_{0} w_{n x}-G_{3, n x}\right), \frac{\varphi_{n x x}}{\lambda_{n}}\right\rangle+k \lambda_{n}\left\|\varphi_{n x}\right\|^{2}+k\left\langle\lambda_{n}\left(\psi_{n}+w_{n}\right), \varphi_{n x}\right\rangle \rightarrow 0 ;
\end{aligned}
$$

hence, using (5.4) 1 , (5.6), (5.13), (5.15), (5.17), (5.18) and (5.47), we obtain (5.50). Therefore, according to the boundary conditions, (5.50) leads to (5.51). Therefore, (5.52), (5.53) and (5.54) hold using the same proof as in Section 5.2.

Integrating by parts and using the boundary conditions, we have

$$
\begin{aligned}
\lambda_{n}\left\langle\left(\varphi_{n x}+\psi_{n}+w_{n}\right)_{x}, \tilde{\varphi}_{n}\right\rangle= & -\lambda_{n}\left\langle\left(\varphi_{n x}+\psi_{n}+w_{n}\right), \tilde{\varphi}_{n x}\right\rangle \\
= & -\frac{1}{k}\left\langle\lambda_{n}^{2}\left[i \lambda_{n} \rho_{3} \tilde{w}_{n}-k_{0} \psi_{n x x}+k\left(\varphi_{n x}+\psi_{n}+w_{n}\right)+\delta w_{n}+G_{3, n x x}\right], \frac{\tilde{\varphi}_{n x}}{\lambda_{n}}\right\rangle \\
& +\frac{\delta}{k}\left\langle w_{n}, \frac{\tilde{\varphi}_{n x}}{\lambda_{n}}\right\rangle-\frac{1}{k}\left\langle i \lambda_{n} \rho_{3} \tilde{w}_{n}, \lambda_{n}\left(i \lambda_{n} \varphi_{n x}-\tilde{\varphi}_{n x}\right)\right\rangle \\
& +\frac{1}{k}\left\langle\left|\lambda_{n}\right|^{\frac{5}{2}}\left(k_{0} \psi_{n x}-G_{3, n x}\right), \frac{i \varphi_{n x x}}{\left|\lambda_{n}\right|^{\frac{1}{2}}}\right\rangle \\
& +\frac{1}{k}\left\langle\left(k_{0} w_{n x x}-G_{3, n x x}\right), \lambda_{n}\left(i \lambda_{n} \varphi_{n x}-\tilde{\varphi}_{n x}\right)\right\rangle \\
& -\frac{\lambda_{n}^{3}}{k\left|\lambda_{n}\right|^{3}}\left\langle i \rho_{3}\left|\lambda_{n}\right|^{\frac{3}{2}} \tilde{w}_{n x}, i\left|\lambda_{n}\right|^{\frac{3}{2}} \varphi_{n}\right\rangle,
\end{aligned}
$$

then, using $(5.4)_{1},(5.4)_{6},(5.6),(5.13),(5.15),(5.16),(5.48),(5.53),(5.54)$ and (5.61), we find (5.55), and therefore (5.56), (5.57) and (5.58) are valid (as in Section 5.2).

Taking the inner product of $(5.4)_{2}$ with $\frac{\psi_{n x}}{\lambda_{n}^{10}}$ in $L^{2}(0,1)$ and using (4.5) and (4.6), we get

$$
\rho_{1}\left\langle i \lambda_{n} \tilde{\varphi}_{n}, \psi_{n x}\right\rangle-k\left\langle\varphi_{n x x}, \psi_{n x}\right\rangle-k\left\|\psi_{n x}\right\|^{2}-k\left\langle w_{n x}, \psi_{n x}\right\rangle \rightarrow 0
$$

then, integrating by parts and using the boundary conditions, we obtain

$$
\rho_{1}\left\langle i \lambda_{n} \tilde{\varphi}_{n}, \psi_{n x}\right\rangle+k\left\langle\lambda_{n} \varphi_{n x}, \frac{\psi_{n x x}}{\lambda_{n}}\right\rangle-k\left\|\psi_{n x}\right\|^{2}-k\left\langle w_{n x}, \psi_{n x}\right\rangle \rightarrow 0
$$

so, using (5.13), (5.18), (5.56) and (5.58), we deduce that

$$
\psi_{n x} \longrightarrow 0 \text { in } L^{2}(0,1)
$$


Taking the inner product of $(5.4)_{4}$ with $\frac{\psi_{n}}{\lambda_{n}^{10}}$ in $L^{2}(0,1)$, integrating by parts and using (4.5) and (4.6) and the boundary conditions, we get

$$
-\rho_{2}\left\langle\tilde{\psi}_{n},\left(i \lambda_{n} \psi_{n}-\tilde{\psi}_{n}\right)\right\rangle-\rho_{2}\left\|\tilde{\psi}_{n}\right\|^{2}+b\left\|w_{n x}\right\|^{2}+\left\langle k\left(\varphi_{n x}+\psi_{n}+w_{n}\right), \psi_{n}\right\rangle \longrightarrow 0
$$

hence, using (4.25), (5.4) 3 and (5.62), we get

$$
\tilde{\psi}_{n} \longrightarrow 0 \text { in } L^{2}(0,1)
$$

The limits (4.25), (5.6), (5.13), (5.16), (5.47), (5.52), (5.62) and (5.63) imply (4.10), which is a contradiction with (4.5). Consequently, (5.2) holds, and so (5.1) is satisfied in case (2.4) with $j=10$.

Now, the proof of Theorem 5.1 is completed.

\section{Lack of exponential stability}

We prove in this section that (2.12) is not exponentially stable in case (2.2) independently of the parameters, and in cases (2.3) and (2.4) if (4.1) is not satisfied. Namely, we have the following theorem:

Theorem 6.1 We assume that $\mathbf{( H )}$ holds. Then the semigroup associated with (2.12) in case (2.2) is not exponentially stable. However, the semigroup associated with (2.12) in cases (2.3) and (2.4) is not exponentially stable if moreover (4.1) does not hold.

Proof. We use Theorem 2.2 by proving that the second condition in (2.39) is not satisfied. We have to prove that there exists a sequence $\left(\lambda_{n}\right)_{n} \subset \mathbb{R}$ such that

$$
\lim _{n \rightarrow \infty}\left\|\left(i \lambda_{n} I-\mathcal{A}\right)^{-1}\right\| \mathcal{L}(\mathcal{H})=\infty
$$

which is equivalent to find a sequence $\left(F_{n}\right)_{n} \subset \mathcal{H}$ satisfying

$$
\left\|F_{n}\right\|_{\mathcal{H}} \leq 1, \quad n \in \mathbb{N}
$$

and

$$
\lim _{n \rightarrow \infty}\left\|\left(i \lambda_{n} I-\mathcal{A}\right)^{-1} F_{n}\right\|_{\mathcal{H}}=\infty
$$

For this purpose, let

$$
U_{n}=\left(i \lambda_{n} I-\mathcal{A}\right)^{-1} F_{n}, \quad n \in \mathbb{N} .
$$

Then we have to find sequences $\left(\lambda_{n}\right)_{n} \subset \mathbb{R},\left(F_{n}\right)_{n} \subset \mathcal{H}$ and $\left(U_{n}\right)_{n} \subset D(\mathcal{A})$ satisfying (6.1),

$$
\lim _{n \rightarrow \infty}\left\|U_{n}\right\|_{\mathcal{H}}=\infty \quad \text { and } \quad i \lambda_{n} U_{n}-\mathcal{A} U_{n}=F_{n}, n \in \mathbb{N}
$$

Taking

$$
U_{n}=\left(\varphi_{n}, \tilde{\varphi}_{n}, \psi_{n}, \tilde{\psi}_{n}, w_{n}, \tilde{w}_{n}, \eta_{n}\right) \quad \text { and } \quad F_{n}=\left(f_{1, n}, \cdots, f_{7, n}\right)
$$


Then we have from (2.13)-(2.16) and (6.4) the following system:

$$
\left\{\begin{array}{l}
i \lambda_{n} \varphi_{n}-\tilde{\varphi}_{n}=f_{1, n}, \\
i \rho_{1} \lambda_{n} \tilde{\varphi}_{n}-k\left(\varphi_{n x}+\psi_{n}+w_{n}\right)_{x}+G_{1, n x x}=\rho_{1} f_{2, n}, \\
i \lambda_{n} \psi_{n}-\tilde{\psi}_{n}=f_{3, n}, \\
i \rho_{2} \lambda_{n} \tilde{\psi}_{n}-b \psi_{n x x}+k\left(\varphi_{n x}+\psi_{n}+w_{n}\right)+G_{2, n x x}=\rho_{2} f_{4, n}, \\
i \lambda_{n} w_{n}-\tilde{w}_{n}=f_{5, n}, \\
i \rho_{3} \lambda_{n} \tilde{w}_{n}-k_{0} w_{n x x}+k\left(\varphi_{n x}+\psi_{n}+w_{n}\right)+\delta w_{n}+G_{3, n x x}=\rho_{3} f_{6, n}, \\
i \lambda_{n} \eta_{n}+\eta_{n s}-\tilde{f}_{n}=f_{7, n},
\end{array}\right.
$$

where $\tilde{f}_{n}, G_{1, n}, G_{2, n}$ and $G_{3, n}$ are defined in (4.9), and $\eta_{n}$ is defined in term of $f_{n}$ similarly to $\eta$ in (2.7). To simplify the formulas, we put

$$
N=\frac{(2 n+1) \pi}{2}
$$

Choosing

$$
f_{1, n}=f_{2, n}=f_{3, n}=f_{5, n}=f_{7, n}=0, \quad f_{4, n}(x)=c_{1} \sin (N x) \quad \text { and } \quad f_{6, n}(x)=c_{2} \sin (N x),
$$

where $c_{1}$ and $c_{2}$ are constants satisfying

$$
\rho_{2}\left|c_{1}\right|^{2}+\rho_{3}\left|c_{2}\right|^{2} \leq 1
$$

The choices (6.7) and (6.8) imply that $\left(F_{n}\right)_{n} \subset \mathcal{H}$ and

$\left\|F_{n}\right\|_{\mathcal{H}}^{2}=\rho_{2}\left\|f_{4, n}\right\|^{2}+\rho_{3}\left\|f_{6, n}\right\|^{2}=\left(\rho_{2}\left|c_{1}\right|^{2}+\rho_{3}\left|c_{2}\right|^{2}\right) \int_{0}^{1} \sin ^{2}(N x) \mathrm{d} x \leq \rho_{2}\left|c_{1}\right|^{2}+\rho_{3}\left|c_{2}\right|^{2} \leq 1, n \in \mathbb{N}$,

so (6.1) holds. On the other hand, the system (6.5) becomes

$$
\left\{\begin{array}{l}
\tilde{\varphi}_{n}=i \lambda_{n} \varphi_{n}, \quad \tilde{\psi}_{n}=i \lambda_{n} \psi_{n}, \quad \tilde{w}_{n}=i \lambda_{n} w_{n}, \\
-\rho_{1} \lambda_{n}^{2} \varphi_{n}-k\left(\varphi_{n x}+\psi_{n}+w_{n}\right)_{x}+G_{1, n x x}=0, \\
-\rho_{2} \lambda_{n}^{2} \psi_{n}-b \psi_{n x x}+k\left(\varphi_{n x}+\psi_{n}+w_{n}\right)+G_{2, n x x}=c_{1} \rho_{2} \sin (N x), \\
-\rho_{3} \lambda_{n}^{2} w_{n}-k_{0} w_{n x x}+k\left(\varphi_{n x}+\psi_{n}+w_{n}\right)+\delta w_{n}+G_{3, n x x}=c_{2} \rho_{3} \sin (N x), \\
i \lambda_{n} \eta_{n}+\eta_{n s}-i \lambda_{n} f_{n}=0,
\end{array}\right.
$$


where $f_{n}$ is defined in (4.9). We choose

$$
\left\{\begin{array}{l}
\varphi_{n}(x)=\alpha_{n} \cos (N x), \quad \tilde{\varphi}_{n}(x)=i \alpha_{n} \lambda_{n} \cos (N x), \\
\psi_{n}(x)=\theta_{n} \sin (N x), \quad \tilde{\psi}_{n}(x)=i \theta_{n} \lambda_{n} \sin (N x), \\
w_{n}(x)=\tau_{n} \sin (N x), \quad \tilde{w}_{n}(x)=i \tau_{n} \lambda_{n} \sin (N x) \\
\eta_{n}(x, s)=\left(1-e^{-i \lambda_{n} s}\right) f_{n}(x)
\end{array}\right.
$$

where $\left(\alpha_{n}\right)_{n},\left(\theta_{n}\right)_{n}$ and $\left(\tau_{n}\right)_{n}$ are sequences of constants to be fixed later. These choices guarantee that $\left(U_{n}\right)_{n} \subset D(\mathcal{A})$ and the first three equations and the last one in (6.9) are satisfied. Thus, (6.9) holds if its fourth, fifth and sixth equations are satisfied. Using the definition of $\eta_{n}$ in (6.10), we get

$$
g_{0} f_{n x x}-\int_{0}^{\infty} g \eta_{n x x} \mathrm{~d} s=g_{0} f_{n x x}-\int_{0}^{\infty}\left(1-e^{-i \lambda_{n} s}\right) g(s) f_{n x x} \mathrm{~d} s=\left(\int_{0}^{\infty} g(s) e^{-i \lambda_{n} s} \mathrm{~d} s\right) f_{n x x},
$$

then, from (6.10) and (6.11), we observe that the fourth, fifth and sixth equations in (6.9) are satisfied if

$$
\left\{\begin{array}{l}
{\left[\left(k-\varepsilon_{1} \mu_{n}\right) N^{2}-\rho_{1} \lambda_{n}^{2}\right] \alpha_{n}-k N \theta_{n}-k N \tau_{n}=0,} \\
-k N \alpha_{n}+\left[\left(b-\varepsilon_{2} \mu_{n}\right) N^{2}-\rho_{2} \lambda_{n}^{2}+k\right] \theta_{n}+k \tau_{n}=c_{1} \rho_{2}, \\
-k N \alpha_{n}+k \theta_{n}+\left[\left(k_{0}-\varepsilon_{3} \mu_{n}\right) N^{2}-\rho_{3} \lambda_{n}^{2}+k+\delta\right] \tau_{n}=c_{2} \rho_{3},
\end{array}\right.
$$

where we denote

$$
\mu_{n}=\int_{0}^{\infty} g(s) e^{-i \lambda_{n} s} \mathrm{~d} s
$$

and (according to the definition of $f_{n}, G_{1, n}, G_{2, n}$ and $G_{3, n}$ in (4.9))

$$
\left\{\begin{array}{lll}
\varepsilon_{1}=1 & \text { and } \quad \varepsilon_{2}=\varepsilon_{3}=0 & \text { in case }(2.2) \\
\varepsilon_{2}=1 & \text { and } \quad \varepsilon_{1}=\varepsilon_{3}=0 & \text { in case }(2.3) \\
\varepsilon_{3}=1 & \text { and } \quad \varepsilon_{1}=\varepsilon_{2}=0 & \text { in case }(2.4)
\end{array}\right.
$$

Because $g$ is integrable on $\mathbb{R}_{+}$and $\left|e^{-i \lambda_{n} s}\right|=1, n \in \mathbb{N}$, then $\left(\mu_{n}\right)_{n}$ exists. On the other hand, integrating by parts and using the fact that $g$ is non-increasing and $\lim _{s \rightarrow \infty} g(s)=0$, we get

$$
\begin{aligned}
\left|\mu_{n}\right| & =\left|\frac{1}{i \lambda_{n}}\left(g(0)+\int_{0}^{\infty} g^{\prime}(s) e^{-i \lambda_{n} s} \mathrm{~d} s\right)\right| \\
& \leq \frac{1}{\left|\lambda_{n}\right|}\left(g(0)+\int_{0}^{\infty}\left|g^{\prime}(s)\right| \mathrm{d} s\right) \\
& \leq \frac{1}{\left|\lambda_{n}\right|}\left(g(0)-\int_{0}^{\infty} g^{\prime}(s) \mathrm{d} s\right) \\
& \leq \frac{2 g(0)}{\left|\lambda_{n}\right|}
\end{aligned}
$$


therefore, if $\left(\lambda_{n}\right)_{n}$ satisfies $\lim _{n \rightarrow \infty}\left|\lambda_{n}\right|=\infty$, then

$$
\lim _{n \rightarrow \infty} \mu_{n}=0
$$

Now, we distinguish the cases (2.2), (2.3) and (2.4).

\section{1. $\operatorname{Case}(2.2)\left(\varepsilon_{1}=1\right.$ and $\varepsilon_{2}=\varepsilon_{3}=0$ in (6.12))}

We distinguish three subcases.

Subcase 1: $\frac{k}{\rho_{1}} \neq \frac{b}{\rho_{2}}$. Let us choose

$$
\lambda_{n}=\sqrt{\frac{b}{\rho_{2}}} N
$$

and $c_{1}$ and $c_{2}$ such that

$$
c_{2}=\frac{\rho_{2}}{\rho_{3}} c_{1} \text { and } \quad 0<\left|c_{1}\right| \leq \sqrt{\frac{\rho_{3}}{\rho_{2}\left(\rho_{2}+\rho_{3}\right)}},
$$

so (6.8) holds. Thanks to these choices, we see that (6.12) is satisfied for

$$
\tau_{n}=0, \quad \theta_{n}=c_{1} \rho_{2}\left(\frac{1}{k}-\frac{\rho_{2}}{\rho_{1} b+\rho_{2} \mu_{n}}\right) \quad \text { and } \quad \alpha_{n}=\frac{-c_{1} \rho_{2}^{2}}{\left(\rho_{1} b+\rho_{2} \mu_{n}\right) N} .
$$

Because $\frac{k}{\rho_{1}} \neq \frac{b}{\rho_{2}}$ and thanks to (6.14) and (6.16), we have

$$
\lim _{n \rightarrow \infty} \theta_{n}=c_{1} \rho_{2}\left(\frac{1}{k}-\frac{\rho_{2}}{\rho_{1} b}\right) \neq 0
$$

Therefore, from the definition of $N$ in (6.6) and $\left(\lambda_{n}\right)_{n}$ in (6.15), we obtain

$$
\lim _{n \rightarrow \infty}\left|\lambda_{n} \theta_{n}\right|=\infty
$$

On the other hand, we have

$$
\begin{aligned}
\left\|U_{n}\right\|_{\mathcal{H}}^{2} \geq \rho_{2}\left\|\tilde{\psi}_{n}\right\|^{2} & =\rho_{2}\left|\lambda_{n}\right|^{2}\left\|\psi_{n}\right\|^{2} \geq \rho_{2}\left|\lambda_{n} \theta_{n}\right|^{2} \int_{0}^{1} \sin ^{2}(N x) \mathrm{d} x \\
& =\frac{\rho_{2}}{2}\left|\lambda_{n} \theta_{n}\right|^{2} \int_{0}^{1}[1-\cos (2 N x)] \mathrm{d} x \\
& =\frac{\rho_{2}}{2}\left|\lambda_{n} \theta_{n}\right|^{2} .
\end{aligned}
$$

Then (6.19) leads to the limit in (6.4). Consequently, there exist sequences $\left(F_{n}\right)_{n} \subset \mathcal{H}$ and $\left(\lambda_{n}\right)_{n} \subset \mathbb{R}$ satisfying (6.1) and (6.2). Hence, Theorem 2.2 implies that (2.12) is not exponentially stable in case (2.2) with $\frac{k}{\rho_{1}} \neq \frac{b}{\rho_{2}}$. 
Subcase 2: $\frac{k}{\rho_{1}} \neq \frac{k_{0}}{\rho_{3}}$. Let us set

$$
\lambda_{n}=\sqrt{\frac{k_{0}}{\rho_{3}} N^{2}+\frac{\delta}{\rho_{3}}}
$$

and $c_{1}$ and $c_{2}$ as in (6.16). Then (6.12) is satisfied for

$$
\theta_{n}=0, \quad \tau_{n}=c_{1} \rho_{2}\left(\frac{1}{k}-\frac{\rho_{3}}{\rho_{1} k_{0}+\frac{\rho_{1} \delta}{N^{2}}+\rho_{3} \mu_{n}}\right) \quad \text { and } \quad \alpha_{n}=\frac{-c_{1} \rho_{2} \rho_{3}}{\left(\rho_{1} k_{0}+\frac{\rho_{1} \delta}{N^{2}}+\rho_{3} \mu_{n}\right) N} .
$$

According to (6.14), (6.16) and the definition of $N$ in (6.6), we get

$$
\lim _{n \rightarrow \infty} \tau_{n}=c_{1} \rho_{2}\left(\frac{1}{k}-\frac{\rho_{3}}{\rho_{1} k_{0}}\right) \neq 0
$$

since $\frac{k}{\rho_{1}} \neq \frac{k_{0}}{\rho_{3}}$, and then

$$
\lim _{n \rightarrow \infty}\left|\lambda_{n} \tau_{n}\right|=\infty
$$

On the other hand, as in the previous subcase 1 , we have

$$
\left\|U_{n}\right\|_{\mathcal{H}}^{2} \geq \rho_{3}\left\|\tilde{w}_{n}\right\|^{2}=\rho_{3}\left|\lambda_{n}\right|^{2}\left\|w_{n}\right\|^{2}=\rho_{3}\left|\lambda_{n} \tau_{n}\right|^{2} \int_{0}^{1} \sin ^{2}(N x) \mathrm{d} x=\frac{\rho_{3}}{2}\left|\lambda_{n} \tau_{n}\right|^{2} .
$$

Then (6.22) implies the limit in (6.4). Finally, we conclude that (2.12) is not exponentially stable in case (2.2) with $\frac{k}{\rho_{1}} \neq \frac{k_{0}}{\rho_{3}}$.

Subcase 3: $\frac{k}{\rho_{1}}=\frac{b}{\rho_{2}}=\frac{k_{0}}{\rho_{3}}$. Let's take

$$
\lambda_{n}=\sqrt{\frac{k}{\rho_{1}}} N
$$

and $c_{1}$ and $c_{2}$ such that

$$
c_{2}=\frac{\rho_{2}(\delta+1)}{\rho_{3}} c_{1} \quad \text { and } \quad 0<\left|c_{1}\right| \leq \sqrt{\frac{\rho_{3}}{\rho_{2}\left((\delta+1)^{2} \rho_{2}+\rho_{3}\right)}},
$$

which gives (6.8). These choices imply that (6.12) is satisfied for

$$
\tau_{n}=c_{1} \rho_{2}, \quad \theta_{n}=c_{1} \rho_{2}\left(\frac{1}{k}-\frac{1}{k+\mu_{n}}-1\right) \quad \text { and } \quad \alpha_{n}=\frac{-c_{1} \rho_{2}}{\left(k+\mu_{n}\right) N} .
$$

It is clear that (6.22) is valid, and so the proof can be carried out as in subcase 2 .

Finally, (2.12) in case (2.2) is not exponentially stable independently of the values of $k, b, k_{0}, \rho_{1}, \rho_{2}$ and $\rho_{3}$. 
6.2. $\operatorname{Case}(2.3)\left(\varepsilon_{2}=1\right.$ and $\varepsilon_{1}=\varepsilon_{3}=0$ in (6.12))

We have assumed that (4.1) does not hold, so we again distinguish the two subcases $\frac{k}{\rho_{1}} \neq \frac{k_{0}}{\rho_{3}}$ and $\left[\frac{k}{\rho_{1}}=\frac{k_{0}}{\rho_{3}}\right.$ and $\left.\frac{k}{\rho_{1}} \neq \frac{b}{\rho_{2}}\right]$.

Subcase 1: $\frac{k}{\rho_{1}} \neq \frac{k_{0}}{\rho_{3}}$. The proof is identical to the one given in Section 6.1 case 2 by considering the same choices of $\lambda_{n}, c_{1}$ and $c_{2}$, so (6.12) is satisfied for $\alpha_{n}, \theta_{n}$ and $\tau_{n}$ defined in (6.20) with $\mu_{n}$ replaced by 0 , and then (6.21) and (6.22) hold.

Subcase 2: $\frac{k}{\rho_{1}}=\frac{k_{0}}{\rho_{3}}$ and $\frac{k}{\rho_{1}} \neq \frac{b}{\rho_{2}}$. We choose

$$
\lambda_{n}=\sqrt{\frac{k}{\rho_{1}} N^{2}+\frac{k}{\sqrt{\rho_{1} \rho_{3}}} N+\frac{\delta}{\rho_{3}}}, \quad c_{1}=0
$$

and $c_{2}$ such that

$$
0<\left|c_{2}\right| \leq \frac{1}{\sqrt{\rho_{3}}}
$$

in order to get (6.8). These choices show that $(6.12)_{1}$ and $(6.12)_{3}$ are satisfied for

$$
\tau_{n}=-\left(\sqrt{\frac{\rho_{1}}{\rho_{3}}}+\frac{\rho_{1} \delta}{\rho_{3} k N}\right) \alpha_{n}-\theta_{n} \quad \text { and } \quad \theta_{n}=\frac{\rho_{1}}{\rho_{3} k}\left(\frac{\delta}{N^{2}} \sqrt{\frac{\rho_{1}}{\rho_{3}}}+\frac{k-\delta}{N}\right) \alpha_{n}+\frac{c_{2} \sqrt{\rho_{1} \rho_{2}}}{k N},
$$

and $(6.12)_{2}$ holds if moreover $\alpha_{n}=\frac{a_{1}}{a_{2}}$, where

$$
a_{1}=\frac{c_{2} \sqrt{\rho_{1} \rho_{3}}}{k}\left(b-\frac{\rho_{2} k}{\rho_{1}}-\mu_{n}-\frac{\rho_{2} k}{\sqrt{\rho_{1} \rho_{3}} N}-\frac{\rho_{2} \delta}{\rho_{3} N^{2}}\right)
$$

and

$$
a_{2}=k+\frac{k}{N} \sqrt{\frac{\rho_{1}}{\rho_{3}}}+\frac{\rho_{1} \delta}{\rho_{3} N^{2}}+\frac{\rho_{1}}{\rho_{3} k}\left(\frac{\delta}{N} \sqrt{\frac{\rho_{1}}{\rho_{3}}}+k-\delta\right)\left(\frac{\rho_{2} k}{\rho_{1}}-b+\mu_{n}+\frac{\rho_{2} k}{\sqrt{\rho_{1} \rho_{3}} N}+\frac{\rho_{2} \delta}{\rho_{3} N^{2}}\right) .
$$

Using (6.14), (6.26) and the definition of $N$ in (6.6), we obtain

$$
\lim _{n \rightarrow \infty}\left|\alpha_{n}\right|=\frac{\left|c_{2}\right| \sqrt{\rho_{1} \rho_{3}}\left|\frac{\rho_{2} k}{\rho_{1}}-b\right|}{|\tilde{k}|} \quad \text { if } \quad \tilde{k}:=k^{2}+\frac{\rho_{1}}{\rho_{3}}(k-\delta)\left(\frac{\rho_{2} k}{\rho_{1}}-b\right) \neq 0
$$

and

$$
\lim _{n \rightarrow \infty}\left|\alpha_{n}\right|=\infty \quad \text { if } \quad \tilde{k}=0 .
$$

So, because $\frac{k}{\rho_{1}} \neq \frac{b}{\rho_{2}}$, we conclude from the definition of $\left(\lambda_{n}\right)_{n}$ in (6.25) that, in both cases $\tilde{k}=0$ and $\tilde{k} \neq 0$,

$$
\lim _{n \rightarrow \infty}\left|\lambda_{n} \alpha_{n}\right|=\infty
$$


On the other hand, we have

$$
\begin{gathered}
\left\|U_{n}\right\|_{\mathcal{H}}^{2} \geq \rho_{1}\left\|\tilde{\varphi}_{n}\right\|^{2}=\rho_{1}\left|\lambda_{n}\right|^{2}\left\|\varphi_{n}\right\|^{2} \\
\geq \rho_{1}\left|\lambda_{n} \alpha_{n}\right|^{2} \int_{0}^{1} \cos ^{2}(N x) \mathrm{d} x=\frac{\rho_{1}}{2}\left|\lambda_{n} \alpha_{n}\right|^{2} \int_{0}^{1}[1+\cos (2 N x)] \mathrm{d} x=\frac{\rho_{1}}{2}\left|\lambda_{n} \alpha_{n}\right|^{2} .
\end{gathered}
$$

Therefore, (6.27) leads to the limit in (6.4). Consequently, as in the previous cases, we deduce that (2.12) is not exponentially stable in case (2.3) if (4.1) does not hold.

\subsection{Case (2.4) $\left(\varepsilon_{3}=1\right.$ and $\varepsilon_{1}=\varepsilon_{2}=0$ in (6.12))}

We have assumed that (4.1) does not hold, so we distinguish the two subcases $\frac{k}{\rho_{1}} \neq \frac{b}{\rho_{2}}$ and $\left[\frac{k}{\rho_{1}}=\frac{b}{\rho_{2}}\right.$ and $\left.\frac{k}{\rho_{1}} \neq \frac{k_{0}}{\rho_{3}}\right]$.

Subcase 1: $\frac{k}{\rho_{1}} \neq \frac{b}{\rho_{2}}$. The proof is identical to the one given in Section 6.1 case 1 by considering the same choices of $\lambda_{n}, c_{1}$ and $c_{2}$, so (6.12) is satisfied for $\alpha_{n}, \theta_{n}$ and $\tau_{n}$ given in (6.17) with $\mu_{n}$ replaced by 0 , and then (6.18) and (6.19) hold.

Subcase 2: $\frac{k}{\rho_{1}}=\frac{b}{\rho_{2}}$ and $\frac{k}{\rho_{1}} \neq \frac{k_{0}}{\rho_{3}}$. We consider the choices

$$
\lambda_{n}=\sqrt{\frac{k}{\rho_{1}} N^{2}+\frac{k}{\sqrt{\rho_{1} \rho_{2}}} N+\frac{\delta}{\rho_{3}}}, \quad c_{2}=0
$$

and $c_{1}$ such that

$$
0<\left|c_{1}\right| \leq \frac{1}{\sqrt{\rho_{2}}}
$$

which gives (6.8). These choices imply that $(6.12)_{1}$ and $(6.12)_{3}$ hold for

$$
\theta_{n}=-\left(\sqrt{\frac{\rho_{1}}{\rho_{2}}}+\frac{\rho_{1} \delta}{\rho_{3} k N}\right) \alpha_{n}-\tau_{n} \quad \text { and } \quad \tau_{n}=\frac{k N+k \sqrt{\frac{\rho_{1}}{\rho_{2}}}+\frac{\rho_{1} \delta}{\rho_{3} N}}{\left(k_{0}-\frac{\rho_{3} k}{\rho_{1}}-\mu_{n}\right) N^{2}-\frac{\rho_{3} k}{\sqrt{\rho_{1} \rho_{2}}} N} \alpha_{n}
$$

and $(6.12)_{2}$ is satisfied if, in addition,

$$
\alpha_{n}=c_{1} \rho_{2}\left[\frac{2 \delta \sqrt{\rho_{1} \rho_{2}}}{\rho_{3}}+\frac{\delta^{2} \rho_{1} \rho_{2}}{k \rho_{3}^{2} N}+\frac{\left(k \sqrt{\frac{\rho_{2}}{\rho_{1}}}+\frac{k}{N}+\frac{\rho_{2} \delta}{\rho_{3} N}\right)\left(k+\frac{k}{N} \sqrt{\frac{\rho_{1}}{\rho_{2}}}+\frac{\rho_{1} \delta}{\rho_{3} N^{2}}\right)}{k_{0}-\frac{\rho_{3} k}{\rho_{1}}-\mu_{n}-\frac{\rho_{3} k}{N \sqrt{\rho_{1} \rho_{2}}}}\right]^{-1} .
$$

Using (6.14), (6.29) and the definition of $N$ in (6.6), we find

$$
\lim _{n \rightarrow \infty}\left|\alpha_{n}\right|=\frac{\left|c_{1}\right|}{|\tilde{k}|} \sqrt{\frac{\rho_{2}}{\rho_{1}}} \quad \text { if } \quad \tilde{k}:=\frac{k^{2}}{\rho_{1} k_{0}-\rho_{3} k}+\frac{2 \delta}{\rho_{3}} \neq 0
$$


and

$$
\lim _{n \rightarrow \infty}\left|\alpha_{n}\right|=\infty \quad \text { if } \quad \tilde{k}=0
$$

( $\tilde{k}$ has a meaning because $\rho_{1} k_{0}-\rho_{3} k \neq 0$ ). So, in both cases $\tilde{k}=0$ and $\tilde{k} \neq 0$, (6.27) is valid. Consequently, as in the Section 6.2 case 2, we deduce that (2.12) is not exponentially stable in case (2.4) if (4.1) does not hold. The proof of Theorem 6.1 is now completed.

\section{Frictional damping case}

Our results of Sections 2-6 also hold when the infinite memory is replaced by a frictional damping, that is when $\gamma f_{t}$ with $\gamma>0$ is considered instead of the infinite integral in (2.1), where $f$ is defined in (2.8). More precisely, we consider (2.1) with

$$
F_{2}=F_{3}=0 \quad \text { and } \quad F_{1}=\gamma \varphi_{t}
$$

or

$$
F_{1}=F_{3}=0 \quad \text { and } \quad F_{2}=\gamma \psi_{t}
$$

or

$$
F_{1}=F_{2}=0 \quad \text { and } \quad F_{3}=\gamma w_{t}
$$

with the boundary conditions (2.5) and the initial data

$$
\begin{cases}(\varphi, \psi, w)(x, 0)=\left(\varphi_{0}, \psi_{0}, w_{0}\right)(x), & x \in(0,1) \\ \left(\varphi_{t}, \psi_{t}, w_{t}\right)(x, 0)=\left(\varphi_{1}, \psi_{1}, w_{1}\right)(x), & x \in(0,1)\end{cases}
$$

We give here a brief idea of the proof which is one given in Sections 2-6.

\subsection{Well-posedness}

As in Section 2, let



and

$$
\left\{\begin{array}{l}
U=(\varphi, \tilde{\varphi}, \psi, \tilde{\psi}, w, \tilde{w}) \\
U_{0}=\left(\varphi_{0}, \varphi_{1}, \psi_{0}, \psi_{1}, w_{0}, w_{1}\right)
\end{array}\right.
$$


System (2.1) in cases (7.1), (7.2) and (7.3) with boundary conditions (2.5) and initial data (7.4) can be written in the form (2.12), where

$$
\mathcal{A} U=\left(\begin{array}{c}
\tilde{\varphi} \\
\frac{k}{\rho_{1}}\left(\varphi_{x}+\psi+w\right)_{x} \\
\tilde{\psi} \\
\frac{1}{\rho_{2}}\left[b \psi_{x x}-k\left(\varphi_{x}+\psi+w\right)\right] \\
\tilde{w} \\
\frac{1}{\rho_{3}}\left[k_{0} w_{x x}-k\left(\varphi_{x}+\psi+w\right)-\delta w\right]
\end{array}\right)+\mathcal{B} U
$$

and

$$
\mathcal{B} U=\left(0,-\frac{\gamma}{\rho_{1}} \tilde{\varphi}, 0,0,0,0\right)^{T}
$$

in case (7.1),

$$
\mathcal{B} U=\left(0,0,0,-\frac{\gamma}{\rho_{2}} \tilde{\psi}, 0,0\right)^{T}
$$

in case (7.2) and

$$
\mathcal{B} U=\left(0,0,0,0,0,-\frac{\gamma}{\rho_{3}} \tilde{w}\right)^{T}
$$

in case (7.3). We consider the energy Hilbert space $\left(H_{1}\right.$ and $H_{0}$ are defined in (2.17))

$$
\mathcal{H}=H_{1} \times L^{2}(0,1) \times H_{0} \times L^{2}(0,1) \times H_{0} \times L^{2}(0,1)
$$

equipped with the inner product, for any

$$
\begin{aligned}
U_{1}= & \left(\varphi_{1}, \tilde{\varphi}_{1}, \psi_{1}, \tilde{\psi}_{1}, w_{1}, \tilde{w}_{1}\right), U_{2}=\left(\varphi_{2}, \tilde{\varphi}_{2}, \psi_{2}, \tilde{\psi}_{2}, w_{2}, \tilde{w}_{2}\right) \in \mathcal{H}, \\
\left\langle U_{1}, U_{2}\right\rangle_{\mathcal{H}}= & k\left\langle\left(\varphi_{1 x}+\psi_{1}+w_{1}\right),\left(\varphi_{2 x}+\psi_{2}+w_{2}\right)\right\rangle+b\left\langle\psi_{1 x}, \psi_{1 x}\right\rangle+k_{0}\left\langle w_{1 x}, w_{2 x}\right\rangle \\
& +\delta\left\langle w_{1}, w_{2}\right\rangle+\rho_{1}\left\langle\tilde{\varphi}_{1}, \tilde{\varphi}_{2}\right\rangle+\rho_{2}\left\langle\tilde{\psi}_{1}, \tilde{\psi}_{2}\right\rangle+\rho_{3}\left\langle\tilde{w}_{1}, \tilde{w}_{2}\right\rangle .
\end{aligned}
$$

The domain $D(\mathcal{A})$ of $\mathcal{A}$ is given by

$$
D(\mathcal{A})=\left\{U \in \mathcal{H}, \mathcal{A} U \in \mathcal{H}, \varphi_{x}(0)=\psi_{x}(1)=w_{x}(1)=0\right\} .
$$

The well-posedness results are given in the following theorem:

Theorem 7.1 For any $m \in \mathbb{N}$ and $U_{0} \in D\left(\mathcal{A}^{m}\right)$, system (2.12) admits a unique solution $U$ satisfying (2.27). 
Proof. As in Section 2, it is sufficient to prove that $\mathcal{A}$ is dissipative and $0 \in \rho(\mathcal{A})$. First, direct calculations give

$$
\langle\mathcal{A} U, U\rangle_{\mathcal{H}}=-\gamma\|\tilde{f}\|^{2} \leq 0,
$$

where $\tilde{f}$ is defined in (7.5).

Second, let $F=\left(f_{1}, \cdots, f_{6}\right) \in \mathcal{H}$. We prove that there exists $U \in D(\mathcal{A})$ satisfying (2.29). Let us consider the case (7.2). The other two cases (7.1) and (7.3) can be treated exactly in the same way. The first, third and fifth equations in (2.29) are equivalent to (2.30), and then (2.31) holds. Therefore, from (2.30), we see that the second, fourth and sixth equations in (2.29) are reduced to

$$
\left\{\begin{array}{l}
k\left(\varphi_{x}+\psi+w\right)_{x}=\rho_{1} f_{2}, \\
b \psi_{x x}-k\left(\varphi_{x}+\psi+w\right)=\rho_{2} f_{4}+\gamma f_{3}, \\
k_{0} w_{x x}-k\left(\varphi_{x}+\psi+w\right)-\delta w=\rho_{3} f_{6} .
\end{array}\right.
$$

Finally, (2.29) has a solution $U \in D(\mathcal{A})$ if and only if (7.11) admits a solution $(\varphi, \psi, w)$ satisfying (2.36), (2.37) and

$$
\varphi_{x x}, \psi_{x x}, w_{x x} \in L^{2}(0,1) .
$$

By considering the variational formulation of (7.11) in $H_{1} \times H_{0} \times H_{0}$ and using Lax-Milgram theorem and classical elliptic regularity arguments, it follows that (7.11) admits a unique solution $(\varphi, \psi, w)$ satisfying (2.36), (2.37) and (7.12). This proves that (2.29) has a unique solution $U \in D(\mathcal{A})$. Thus, $0 \in \rho(\mathcal{A})$.

Consequently, we conclude that $\mathcal{A}$ is the infinitesimal generator of a linear $C_{0}$ semigroup of contractions on $\mathcal{H}$ and $D(\mathcal{A})$ is dense in $\mathcal{H}$. So, Theorem 7.1 holds.

\subsection{Strong stability}

In this subsection, we prove that (3.1) holds also in cases (7.1), (7.2) and (7.3). More precisely, we have the following result:

Theorem 7.2 For any $U_{0} \in \mathcal{H}$, the solution $U$ of (2.12) in cases (7.2) and (7.3) satisfies (3.1). However, (3.1) holds in case (7.1) if (3.2) is satisfied.

Proof. As in Section 3, to get (3.1), we only have to prove (3.4). We have proved (3.4) for $\lambda=0$ in Section 7.1. So let $\lambda \in \mathbb{R}^{*}$ and

$$
U=(\varphi, \tilde{\varphi}, \psi, \tilde{\psi}, w, \tilde{w}) \in D(\mathcal{A})
$$

satisfying (3.5). From (7.10), we have

$$
0=\operatorname{Re} i \lambda\|U\|_{\mathcal{H}}^{2}=\operatorname{Re}\langle i \lambda U, U\rangle_{\mathcal{H}}=\operatorname{Re}\langle\mathcal{A} U, U\rangle_{\mathcal{H}}=-\gamma\|\tilde{f}\|^{2} .
$$

This leads to (3.7). Consequently, the proof can be carried out as in Section 3.

\subsection{Exponential stability}

In this section, we establish the following exponential stability result: 
Theorem 7.3 We assume that (4.1) holds. Then (2.12) in cases (7.2) and (7.3) is exponentially stable.

Proof. As in Section 4, we will apply Theorem 2.2 by proving (2.39). Condition (4.3) has already been proved in Section 7.2. So we only need to prove (4.4). Assume that (4.4) does not hold, then there exist sequences $\left(U_{n}\right)_{n} \subset D(\mathcal{A})$ and $\left(\lambda_{n}\right)_{n} \subset \mathbb{R}$ satisfying (4.5), (4.6) and (4.7). Let $U_{n}=$ $\left(\varphi_{n}, \tilde{\varphi}_{n}, \psi_{n}, \tilde{\psi}_{n}, w_{n}, \tilde{w}_{n}\right)$. The limit (4.7) implies that

$$
\left\{\begin{array}{l}
i \lambda_{n} \varphi_{n}-\tilde{\varphi}_{n} \rightarrow 0 \text { in } H_{1}, \\
i \lambda_{n} \rho_{1} \tilde{\varphi}_{n}-k\left(\varphi_{n x}+\psi_{n}+w_{n}\right)_{x}+\varepsilon_{1} \gamma \tilde{\varphi}_{n} \rightarrow 0 \text { in } L^{2}(0,1), \\
i \lambda_{n} \psi_{n}-\tilde{\psi}_{n} \rightarrow 0 \text { in } H_{0}, \\
i \lambda_{n} \rho_{2} \tilde{\psi}_{n}-b \psi_{n x x}+k\left(\varphi_{n x}+\psi_{n}+w_{n}\right)+\varepsilon_{2} \gamma \tilde{\psi}_{n} \rightarrow 0 \text { in } L^{2}(0,1), \\
i \lambda_{n} w_{n}-\tilde{w}_{n} \rightarrow 0 \text { in } H_{0}, \\
i \lambda_{n} \rho_{3} \tilde{w}_{n}-k_{0} w_{n x x}+k\left(\varphi_{n x}+\psi_{n}+w_{n}\right)+\delta w_{n}+\varepsilon_{3} \gamma \tilde{w}_{n} \rightarrow 0 \text { in } L^{2}(0,1),
\end{array}\right.
$$

where

$$
\left\{\begin{array}{lll}
\varepsilon_{1}=1 \quad \text { and } \quad \varepsilon_{2}=\varepsilon_{3}=0 & \text { in case }(7.1) \\
\varepsilon_{2}=1 \quad \text { and } \quad \varepsilon_{1}=\varepsilon_{3}=0 & \text { in case }(7.2) \\
\varepsilon_{3}=1 \quad \text { and } \quad \varepsilon_{1}=\varepsilon_{2}=0 & \text { in case }(7.3)
\end{array}\right.
$$

We will prove (4.10), which gives a contradiction with (4.5). Using (7.10), we get

$$
\operatorname{Re}\left\langle\left(i \lambda_{n} I-\mathcal{A}\right) U_{n}, U_{n}\right\rangle_{\mathcal{H}}=\operatorname{Re}\left(i \lambda_{n}\left\|U_{n}\right\|_{\mathcal{H}}^{2}-\left\langle\mathcal{A} U_{n}, U_{n}\right\rangle_{\mathcal{H}}\right)=\gamma\left\|\tilde{f}_{n}\right\|^{2}
$$

So (4.5) and (4.7) imply that

$$
\tilde{f}_{n} \longrightarrow 0 \text { in } L^{2}(0,1)
$$

Therefore, from $(7.13)_{1},(7.13)_{3}$ and $(7.13)_{5}$, we get

$$
\lambda_{n} f_{n} \longrightarrow 0 \text { in } L^{2}(0,1)
$$

where $f_{n}$ is defined in (7.5). Multiplying $(7.13)_{2},(7.13)_{4}$ and (7.13) 6 by $\varphi_{n}, \psi_{n}$ and $w_{n}$, respectively, integration by parts and using (4.5), we see that

$$
\begin{gathered}
\left\langle\left(i \rho_{1} \tilde{\varphi}_{n}-\frac{k}{\lambda_{n}} \psi_{n x}-\frac{k}{\lambda_{n}} w_{n x}+\frac{\varepsilon_{1} \gamma}{\lambda_{n}} \tilde{\varphi}_{n}\right), \lambda_{n} \varphi_{n}\right\rangle+k\left\|\varphi_{n x}\right\| \longrightarrow 0, \\
\left\langle\left(i \rho_{2} \tilde{\psi}_{n}+\frac{k}{\lambda_{n}}\left(\varphi_{n x}+\psi_{n}+w_{n}\right)+\frac{\varepsilon_{2} \gamma}{\lambda_{n}} \tilde{\psi}_{n}\right), \lambda_{n} \varphi_{n}\right\rangle+b\left\|\psi_{n x}\right\| \longrightarrow 0
\end{gathered}
$$


and

$$
\left\langle\left(i \rho_{3} \tilde{w}_{n}+\frac{k}{\lambda_{n}}\left(\varphi_{n x}+\psi_{n}+w_{n}\right)+\frac{\varepsilon_{3} \gamma}{\lambda_{n}} \tilde{w}_{n}+\frac{\delta}{\lambda_{n}} w_{n}\right), \lambda_{n} \varphi_{n}\right\rangle+k_{0}\left\|w_{n x}\right\| \longrightarrow 0 .
$$

So, using (4.5), (4.6) and (7.16), we arrive at (4.22). The proof of Theorem 7.3 can be then finalized as in Section 4.

\subsection{Polynomial stability}

In this section, we prove the following improved polynomial decay rate of (2.12) in cases (7.1), (7.2) and (7.3) compared to the one given in (5.1):

Theorem 7.4 For any $m \in \mathbb{N}^{*}$, there exists a constant $c_{m}>0$ such that (5.1) is satisfied in cases (7.2) and (7.3) with $j=8$. However, (5.1) holds in case (7.1) with $j=2$ if (3.2) is satisfied.

Proof. Using Theorem 2.3, we need to show (4.3) and (5.2), where $j=2$ in case (7.1) and $j=8$ in cases (7.2) and (7.3). Condition (4.3) was proved in Section 7.2. As in Section 5, we establish (5.2) by contradiction. Assume that (5.2) is false, then there exist sequences $\left(U_{n}\right)_{n} \subset D(\mathcal{A})$ and $\left(\lambda_{n}\right)_{n} \subset \mathbb{R}$ satisfying (4.5), (4.6) and (5.3). Let $U_{n}=\left(\varphi_{n}, \tilde{\varphi}_{n}, \psi_{n}, \tilde{\psi}_{n}, w_{n}, \tilde{w}_{n}\right)$. The limit (5.3) implies that

$$
\left\{\begin{array}{l}
\lambda_{n}^{j}\left[i \lambda_{n} \varphi_{n}-\tilde{\varphi}_{n}\right] \rightarrow 0 \text { in } H_{1} \\
\lambda_{n}^{j}\left[i \lambda_{n} \rho_{1} \tilde{\varphi}_{n}-k\left(\varphi_{n x}+\psi_{n}+w_{n}\right)_{x}+\varepsilon_{1} \gamma \tilde{\varphi}_{n}\right] \rightarrow 0 \text { in } L^{2}(0,1) \\
\lambda_{n}^{j}\left[i \lambda_{n} \psi_{n}-\tilde{\psi}_{n}\right] \rightarrow 0 \text { in } H_{0} \\
\lambda_{n}^{j}\left[i \lambda_{n} \rho_{2} \tilde{\psi}_{n}-b \psi_{n x x}+k\left(\varphi_{n x}+\psi_{n}+w_{n}\right)+\varepsilon_{2} \gamma \tilde{\psi}_{n}\right] \rightarrow 0 \text { in } L^{2}(0,1) \\
\lambda_{n}^{j}\left[i \lambda_{n} w_{n}-\tilde{w}_{n}\right] \rightarrow 0 \text { in } H_{0}, \\
\lambda_{n}^{j}\left[i \lambda_{n} \rho_{3} \tilde{w}_{n}-k_{0} w_{n x x}+k\left(\varphi_{n x}+\psi_{n}+w_{n}\right)+\delta w_{n}+\varepsilon_{3} \gamma \tilde{w}_{n}\right] \rightarrow 0 \text { in } L^{2}(0,1)
\end{array}\right.
$$

where $\varepsilon_{1}, \varepsilon_{2}$ and $\varepsilon_{3}$ are defined in (7.14). We will prove (4.10), which gives a contradiction with (4.5). Using (7.10), we get

$$
\operatorname{Re}\left\langle\lambda_{n}^{j}\left(i \lambda_{n} I-\mathcal{A}\right) U_{n}, U_{n}\right\rangle_{\mathcal{H}}=\operatorname{Re}\left(i \lambda_{n}^{j+1}\left\|U_{n}\right\|_{\mathcal{H}}^{2}-\lambda_{n}^{j}\left\langle\mathcal{A} U_{n}, U_{n}\right\rangle_{\mathcal{H}}\right)=\gamma \lambda_{n}^{j}\left\|\tilde{f}_{n}\right\|^{2}
$$

So (4.5) and (5.3) imply that

$$
\lambda_{n}^{\frac{j}{2}} \tilde{f}_{n} \longrightarrow 0 \text { in } L^{2}(0,1)
$$

Multiplying $(7.17)_{1},(7.17)_{3}$ and (5.4) $)_{5}$ by $\left|\lambda_{n}\right|^{-\frac{j}{2}}$ and using (4.6) and (7.18), we get

$$
\lambda_{n}^{\frac{j}{2}+1} f_{n} \longrightarrow 0 \text { in } L^{2}(0,1)
$$


nultiplying $(7.17)_{2},(7.17)_{4}$ and $(7.17)_{6}$ by $\lambda_{n}^{-\frac{j}{2}+1} \varphi_{n}, \lambda_{n}^{-\frac{j}{2}+1} \psi_{n}$ and $\lambda_{n}^{-\frac{j}{2}+1} w_{n}$, respectively, integration by parts and using (4.5) and (4.6), we see that

$$
\begin{gathered}
\left\langle\left(i \lambda_{n} \rho_{1} \tilde{\varphi}_{n}-k \psi_{n x}-k w_{n x}+\varepsilon_{1} \gamma \tilde{\varphi}_{n}\right), \lambda_{n}^{\frac{j}{2}+1} \varphi_{n}\right\rangle+k \lambda_{n}^{\frac{j}{2}+1}\left\|\varphi_{n x}\right\|^{2} \longrightarrow 0, \\
\left\langle\left(i \lambda_{n} \rho_{2} \tilde{\psi}_{n}+k\left(\varphi_{n x}+\psi_{n}+w_{n}\right)+\varepsilon_{2} \gamma \tilde{\psi}_{n}\right), \lambda_{n}^{\frac{j}{2}+1} \psi_{n}\right\rangle+b \lambda_{n}^{\frac{j}{2}+1}\left\|\psi_{n x}\right\|^{2} \longrightarrow 0
\end{gathered}
$$

and

$$
\left\langle\left(i \lambda_{n} \rho_{3} \tilde{w}_{n}+k\left(\varphi_{n x}+\psi_{n}+w_{n}\right)+\varepsilon_{3} \gamma \tilde{w}_{n}+\delta w_{n}\right), \lambda_{n}^{\frac{j}{2}+1} w_{n}\right\rangle+k_{0} \lambda_{n}^{\frac{j}{2}+1}\left\|w_{n x}\right\|^{2} \longrightarrow 0,
$$

so, using (4.5), (7.18) and (7.19), we get

$$
\left|\lambda_{n}\right|^{\frac{j}{4}+\frac{1}{2}} f_{n x} \longrightarrow 0 \text { in } L^{2}(0,1)
$$

We note that (7.20) with $j=2$ and $j=8$ is identical to (5.13) with $j=4$ and $j=10$, respectively. Now, the proof of Theorem 7.4 can be finished as in Section 5.

\subsection{Lack of exponential stability}

As in Section 6, we have the following theorem:

THEOREM 7.5 The semigroup associated with (2.12) in case (7.1) is not exponentially stable. However, the semigroup associated with (2.12) in cases (7.2) and (7.3) is not exponentially stable if (4.1) does not hold.

Proof. As in Section 6, we use Theorem 2.2 by proving that the second condition in (2.39) is not satisfied, that is we have to find sequences $\left(\lambda_{n}\right)_{n} \subset \mathbb{R}$ and $\left(F_{n}\right)_{n} \subset \mathcal{H}$ such that the sequence $\left(U_{n}\right)_{n}$ defined in (6.3) satisfies (6.4). Taking

$$
U_{n}=\left(\varphi_{n}, \tilde{\varphi}_{n}, \psi_{n}, \tilde{\psi}_{n}, w_{n}, \tilde{w}_{n}\right) \quad \text { and } \quad F_{n}=\left(f_{1, n}, \cdots, f_{6, n}\right)
$$

then we have the following system:

$$
\left\{\begin{array}{l}
i \lambda_{n} \varphi_{n}-\tilde{\varphi}_{n}=f_{1, n}, \\
i \rho_{1} \lambda_{n} \tilde{\varphi}_{n}-k\left(\varphi_{n x}+\psi_{n}+w_{n}\right)_{x}+\varepsilon_{1} \gamma \tilde{\varphi}_{n}=\rho_{1} f_{2, n}, \\
i \lambda_{n} \psi_{n}-\tilde{\psi}_{n}=f_{3, n}, \\
i \rho_{2} \lambda_{n} \tilde{\psi}_{n}-b \psi_{n x x}+k\left(\varphi_{n x}+\psi_{n}+w_{n}\right)+\varepsilon_{2} \gamma \tilde{\psi}_{n}=\rho_{2} f_{4, n}, \\
i \lambda_{n} w_{n}-\tilde{w}_{n}=f_{5, n}, \\
i \rho_{3} \lambda_{n} \tilde{w}_{n}-k_{0} w_{n x x}+k\left(\varphi_{n x}+\psi_{n}+w_{n}\right)+\delta w_{n}+\varepsilon_{3} \gamma \tilde{w}_{n}=\rho_{3} f_{6, n} .
\end{array}\right.
$$


We consider the notation (6.6) and the choices (6.7), (6.8) and (6.10) (without $f_{7, n}$ and $\eta_{n}$ ). Then (7.21) is satisfied if (6.12) holds with $\mu_{n}=\frac{-i \gamma \lambda_{n}}{N^{2}}$ instead of (6.13). So, distinguishing the same cases and using the same choices of $\alpha_{n}, \theta_{n}, \tau_{n}, f_{4, n}, f_{6, n}$ and $\lambda_{n}$ as in Section 6 (so (6.14) is satisfied), Theorem 7.5 follows.

\section{Comments and issues}

1. Our results in cases (2.3), (2.4), (7.2) and (7.3) remain valid for $\delta=0$, as well as the lack of exponential stability result in cases (2.2) and (7.1). However, our strong and polynomial stability results in cases (2.2) and (7.1) remain valid for $\delta=0$ if $\frac{b}{\rho_{2}} \neq \frac{k_{0}}{\rho_{3}}$ (condition (3.2) with $\delta=0)$.

2. Our results hold true for one of the following Dirichlet-Neumann boundary conditions:

$$
\begin{cases}\varphi_{x}(0, t)=\varphi_{x}(1, t)=\psi(0, t)=\psi(1, t)=w(0, t)=w(1, t), & t \in \mathbb{R}_{+}, \\ \varphi(0, t)=\varphi(1, t)=\psi_{x}(0, t)=\psi_{x}(1, t)=w_{x}(0, t)=w_{x}(1, t), & t \in \mathbb{R}_{+}, \\ \varphi(0, t)=\varphi_{x}(1, t)=\psi_{x}(0, t)=\psi(1, t)=w_{x}(0, t)=w(1, t), & t \in \mathbb{R}_{+} .\end{cases}
$$

The question is posed when $(\varphi$ and $\psi)$ or $(\varphi$ and $w)$ have the same boundary conditions at 0 or at 1 and also when $\psi$ and $w$ have different boundary conditions at 0 or at 1 . It will be interesting to consider this kind of boundary conditions, in particular, the homogeneous Dirichlet boundary conditions for all $\varphi, \psi$ and $w$ at 0 and/or at 1 .

3. Proving the stability under only one boundary control would be of great importance.

4. The condition (2.21) is the simplest standard one on the relaxation function $g$. Seeking the largest class possible of kernels was not among the objectives of this paper. But we think that it will be possible to weaken (2.21) and get the strong and polynomial stability (perhaps with smaller decay rates than the ones given in this paper). For this issue, we refer the readers to Guesmia (2014), Guesmia \& Messaoudi (2014) and Guesmia et al. (2012) in case of Timoshenko beams and to Guesmia (2017a), Guesmia \& Kafini (2015) and Guesmia \& Kirane (2016) in case of Bresse system.

5. The infinite memory and the frictional damping lead to the same strong and exponential stability results. However, the obtained polynomial decay rate under the frictional damping is better than the one obtained under the infinite memory. In both infinite memory and frictional damping cases, the decay rates given in (5.1) can be probably improved. In general, when the exponential stability is not satisfied, obtaining the optimal decay rate of solutions is, in our opinion, a very nice and hard question

\section{Acknowledgements}

The author would like to express his gratitude to the anonymous referees for very careful reading and punctual suggestions. 


\section{REFERENCES}

Alves, M. S., Gamboa, P., Gorain, G. C., Rambaud, A. \& Vera, O. (2016) Asymptotic behavior of a flexible structure with Cattaneo type of thermal effect. Indag. Math. (N.S.), 27, 821-834.

ARENDT, W. \& BATTY, C. J. K. (1988) Tauberian theorems and stability of one one-parameter semigroups. Trans. Amer. Math. Soc., 306, 837-852.

Beards, C. F. \& Imam, I. M. A. (1978) The damping of plate vibration by interfacial slip between layers. Int. J. Mach. Tool. Des. Res., 18, 131-137.

Bresse, J. A. C. (1859) Cours de Méchanique Appliquée. Paris: Mallet Bachelier.

CAO, X. G., LiU, D. Y. \& XU, G. Q. (2007) Easy test for stability of laminated beams with structural damping and boundary feedback controls. J. Dyn. Control Syst., 13, 313-336.

Cavalcanti, M. M., Domingos Cavalcanti, V. N., Falcão Nascimento, F. A., Lasiecka, I. \& Rodrigues, J. H. (2014) Uniform decay rates for the energy of Timoshenko system with the arbitrary speeds of propagation and localized nonlinear damping. Z. Angew. Math. Phys., 65, 1189-1206.

Dafermos, C. M. (1970) Asymptotic stability in viscoelasticity. Arch. Ration. Mech. Anal., 37, 297-308.

Guesmia, A. (2014) Some well-posedness and general stability results in Timoshenko systems with infinite memory and distributed time delay. J. Math. Phys., 55, 1-40.

Guesmia, A. (2017a) Asymptotic stability of Bresse system with one infinite memory in the longitudinal displacements. Mediterr. J. Math., 14, 19.

Guesmia, A. (2017b) Non-exponential and polynomial stability results of a Bresse system with one infinite memory in the vertical displacement. Nonauton. Dyn. Syst., 4, 78-97.

Guesmia, A. \& Kafini, M. (2015) Bresse system with infinite memories. Math. Methods Appl. Sci., 38, $2389-2402$.

Guesmia, A. \& Kirane, M. (2016) Uniform and weak stability of Bresse system with two infinite memories. Z. Angew. Math. Phys., 67, 1-39.

Guesmia, A. \& Messaoudi, S. (2014) A general stability result in a Timoshenko system with infinite memory: a new approach. Math. Methods Appl. Sci., 37, 384-392.

Guesmia, A., Messaoudi, S. \& Soufyane, A. (2012) Stabilization for a linear Timoshenko system with infinite history and applications to the coupled Timoshenko-heat systems. Electron. J. Differential Equations, 2012, $1-45$.

Hansen, S. W. (1994) A model for a two-layered plate with interfacial slip. Control and estimation of distributed parameter systems: nonlinear phenomena. International Series of Numerical Analysis, vol. 118. Basel: Birkhauser, pp. 143-170.

Hansen, S. W. \& SpIEs, R. (1997) Structural damping in a laminated beams due to interfacial slip. J. Sound Vib., 204, 183-202.

HUANG, F. L. (1985) Characteristic condition for exponential stability of linear dynamical systems in Hilbert spaces. Ann. Diff. Eqns., 1, 43-56.

LiU, Z. \& RAO, B. (2005) Characterization of polynomial decay rate for the solution of linear evolution equation. Z. Angew. Math. Phys., 56, 630-644.

LiU, W. \& ZhaO, W. (2017) Exponential and polynomial decay for a laminated beam with Fourier's type heat conduction. Preprints, 2017020058. doi: 10.20944/preprints201702.0058.v1.

Liu, Z. \& Zheng, S. (1999) Semigroups Associated With Dissipative Systems. New York: Chapman \& Hall/CRC.

Lo, A. \& TATAR, N. E. (2015) Stabilization of laminated beams with interfacial slip. Electron. J. Differential Equations, 2015, 1-14.

Lo, A. \& TATAR, N. E. (2016a) Uniform stability of a laminated beam with structural memory. Qual. Theory Dyn. Syst., 15, 517-540.

Lo, A. \& Tatar, N. E. (2016b) Exponential stabilization of a structure with interfacial slip. Discrete Contin. Dyn. Syst., 36, 6285-6306.

PAZY, A. (1983) Semigroups of Linear Operators and Applications to Partial Differential Equations. New York: Springer. 
Prüss, J. (1984) On the spectrum of $C_{0}$-semigroups. Trans. Amer. Math. Soc., 284, 847-857.

RAposo, C. A. (2016) Exponential stability for a structure with interfacial slip and frictional damping. Appl. Math. Lett., 53, 85-91.

Raposo, C. A., Villagrán, O. V., Muñoz Rivera, J. E. \& Alves, M. S. (2017) Hybrid laminated Timoshenko beam. J. Math. Phys., 58, 11.

TAtar N. E.. (2015) Stabilization of a laminated beam with interfacial slip by boundary controls. Bound. Value Probl., 2015, 11.

Timoshenko, S. (1921) On the correction for shear of the differential equation for transverse vibrations of prismatic bars. Philis. Mag., 41, 744-746.

WANG, J. M., XU, G. Q. \& Yung, S. P. (2005) Exponential stabilization of laminated beams with structural damping and boundary feedback controls. SIAM J. Control Optim., 44, 1575-1597. 